\title{
Formality Theorems for Hochschild Complexes and Their Applications
}

\author{
VASILIY DOLGUSHEV ${ }^{1}$, DMITRY TAMARKIN ${ }^{2}$ and BORIS TSYGAN ${ }^{2}$ \\ ${ }^{1}$ Department of Mathematics, University of California at Riverside, 900 Big Springs Drive, \\ Riverside, CA 92521, USA. e-mail: vald@math.ucr.edu \\ ${ }^{2}$ Mathematics Department, Northwestern University, 2033 Sheridan Rd., Evanston, \\ IL60208, USA.e-mail: tamarkin@math.northwestern.edu; tsygan@math.northwestern.edu
}

Received: 19 January 2009 / Revised: 19 June 2009 / Accepted: 2 July 2009

Published online: 26 September 2009 - (C) The Author(s) 2009. This article is published with open access at Springerlink.com

\begin{abstract}
We give a popular introduction to formality theorems for Hochschild complexes and their applications. We review some of the recent results and prove that the truncated Hochschild cochain complex of a polynomial algebra is non-formal.
\end{abstract}

Mathematics Subject Classification (2000). 19D55, 53D55.

Keywords. Hochschild complexes, deformation quantization.

\section{Contents}

1 Introduction . . . . . . . . . . . . . . . . . . . . . . . . . . 104

2 Formal Versus Non-Formal . . . . . . . . . . . . . . . . . . . . . 105

2.1 GENERAL DEFINITIONS . . . . . . . . . . . . . . . . . . 105

2.2 FROM $L_{\infty}$ ALGEBRAS BACK TO DG LIE ALGEBRAS . . . . . . . . . . . 109

2.3 DG LIE ALGEBRAS AND MAURER-CARTAN ELEMENTS . . . . . . . . . . 109

2.4 TWISTING BY A MC ELEMENT . . . . . . . . . . . . . . . . . 111

3 Algebraic Structures on the Hochschild Complexes . . . . . . . . . . . . . . 111

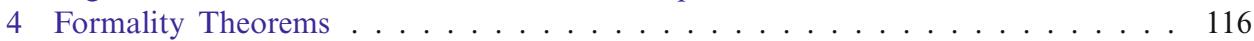

4.1 ALTERNATIVE APPROACH TO THEOREM $4 \ldots \ldots \ldots \ldots \ldots \ldots$

4.2 FORMALITY THEOREMS FOR HOCHSCHILD AND CYCLIC CHAINS . . 119

4.3 FORMALITY OF THE $\infty$-CALCULUS ALGEBRA $\left(C^{\bullet}(A), C_{\bullet}(A)\right) \ldots \ldots . . .121$

4.4 FORMALITY THEOREMS FOR HOCHSCHILD AND CYCLIC COMPLEXES

IN THE ALGEBRAIC GEOMETRY SETTING . . . . . . . . . . . . . . . . 123

5 More Applications of Formality Theorems . . . . . . . . . . . . . . . . . . . 124

6 An Example of a Non-Formal DG Lie Algebra . . . . . . . . . . . . . . . . 126

To Giovanni Felder on the occasion of his 50th birthday. 


\section{Introduction}

The notion of formality was suggested in the classical article [31] of Deligne, Griffiths, Morgan, and Sullivan. In this article it was shown that the de Rham algebra of a compact Kähler manifold $M$ is quasi-isomorphic to the cohomology ring of $M$. Using the terminology suggested in this article we can say that the de Rham algebra of a compact Kähler manifold $M$ is formal as a commutative algebra.

Around 1993-1994 Kontsevich conjectured (see [59,91]) that the complex of continuous Hochschild cochains of the algebra of functions on a smooth manifold is formal as a Lie algebra with the Gerstenhaber bracket [44]. Then in 1997 Kontsevich proved [58] this formality conjecture for an arbitrary smooth manifold. In the same paper he showed how this result solves a long standing problem on the deformation quantization $[7,8,10,11]$ of a Poisson manifold.

In 1998 the second author proposed a completely different proof of Kontsevich's formality theorem [82] for the case of the affine space over an arbitrary field of characteristic zero. This approach is based on deep results such as the proof of Deligne's conjecture on the Hochschild complex [12,62,74,84,92] and the formality theorem $[61,66,83]$ for the operad of little discs [72].

In 1999 Cattaneo and Felder [24] described how Kontsevich's star-product formula as well as his formality theorem can be obtained using the correlators of the Poisson sigma model [56,76].

After Kontsevich's celebrated result [58] many interesting generalizations and applications of the formality theorem for the Hochschild cochain complex were proposed. At this moment all these results can be put under the umbrella of an independent mathematical topic. In this paper we give a popular introduction to this fascinating topic. We review some of the recent results and give an example of a non-formal differential graded (DG) Lie algebra. We hope that our introductory part is accessible to graduate students who are interested in this topic.

The organization of the paper is as follows. In the next section we illustrate the general concept of formality with the example of a DG Lie algebra. We recall Maurer-Cartan elements, the Goldman-Millson groupoid and twisting procedure. We also discuss some consequences of formality for a DG Lie algebra. In Section 3 we recall basic algebraic structures on the Hochschild complexes of an associative algebra $A$. In this section we also recall the Van den Bergh duality theorem [88]. Section 4 begins with the formulation of Kontsevich's formality theorem [58] and its immediate corollaries. Next we review the alternative approach [82] of the second author. Then we discuss formality theorems for Hochschild and cyclic chains and the formality of the $\infty$-calculus algebra of Hochschild complexes. We conclude Section 4 with the formality theorems for Hochschild complexes in the algebraic geometry setting. In Section 5 we give a brief outline of a few recent applications of the formality theorems for Hochschild complexes. Finally, in the concluding section we give an example of a non-formal DG Lie algebra. 
Notation. "DG" stands for differential graded. $S_{n}$ denotes the symmetric group on $n$ letters. By suspension $\mathbf{s} V$ of a graded vector space (or a cochain complex) $V$ we mean $\varepsilon \otimes V$, where $\varepsilon$ is a one-dimensional vector space placed in degree +1 . For a vector $v \in V$ we denote by $|v|$ its degree. ${ }^{1}$ We use the Koszul rule of signs which says that a transposition of any two homogeneous vectors $v_{1}$ and $v_{2}$ yields the sign

$$
(-1)^{\left|v_{1}\right|\left|v_{2}\right|} \text {. }
$$

For a groupoid $\mathcal{G}$ we denote by $\pi_{0}(\mathcal{G})$ the set of isomorphism classes of its objects. This is exactly the set of connected components of the classifying space $B \mathcal{G}$ of $\mathcal{G}$. We assume that the underlying field $\mathbb{K}$ has characteristic zero. $\hbar$ denotes the formal deformation parameter.

\section{Formal Versus Non-Formal}

\subsection{GENERAL DEFINITIONS}

Let $\mathcal{L}$ and $\widetilde{\mathcal{L}}$ be DG Lie algebras over the field $\mathbb{K}$. We say that a morphism $\mu: \mathcal{L} \rightarrow$ $\widetilde{\mathcal{L}}$ is a quasi-isomorphism if $\mu$ induces an isomorphism on cohomology groups. In this case we use the tilde over the arrow

$$
\mu: \mathcal{L} \stackrel{\sim}{\rightarrow} \widetilde{\mathcal{L}} .
$$

We call two DG Lie algebras $\mathcal{L}$ and $\widetilde{\mathcal{L}}$ quasi-isomorphic if they can be connected by a sequence of quasi-isomorphisms

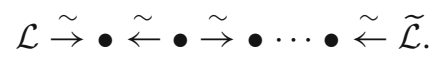

For every DG Lie algebra $\mathcal{L}$, its cohomology $H^{\bullet}(\mathcal{L})$ is naturally a graded Lie algebra. We think of $H^{\bullet}(\mathcal{L})$ as the DG Lie algebra with the zero differential.

DEFINITION 1. A DG Lie algebra $\mathcal{L}$ is called formal if it is quasi-isomorphic to its cohomology $H^{\bullet}(\mathcal{L})$.

Remark. Similarly, we can talk about formal or non-formal DG associative algebras, DG commutative algebras and DG algebras of other types.

We would like to mention that, since our DG Lie algebras are complexes of vector spaces over a field, we always have a map of cochain complexes

$$
F_{1}: H^{\bullet}(\mathcal{L}) \rightarrow \mathcal{L},
$$

which induces an isomorphism on the level of cohomology. In other words, it is always possible to choose a representative for each cohomology class in such a way that this choice respects linearity.

\footnotetext{
${ }^{1}$ For $v \in \mathbf{s} V$ or $v \in \mathbf{s}^{-1} V$ we denote by $|v|$ its degree in the original graded vector space $V$.
} 
It is obvious that, in general, $F_{1}$ is not compatible with the Lie brackets. However, there exists a bilinear map

$$
F_{2}: \wedge^{2} H^{\bullet}(\mathcal{L}) \rightarrow \mathcal{L}
$$

of degree -1 such that

$$
F_{1}\left(\left[v_{1}, v_{2}\right]\right)-\left[F_{1}\left(v_{1}\right), F_{1}\left(v_{2}\right)\right]=\partial F_{2}\left(v_{1}, v_{2}\right),
$$

where $\partial$ is the differential on $\mathcal{L}$.

It is convenient to enlarge the category of DG Lie algebras to by $L_{\infty}$ algebras $[55,65]$. These more general algebras have two important advantages. First, every sequence of quasi-isomorphisms between $L_{\infty}$ algebras $\mathcal{L}$ and $\widetilde{\mathcal{L}}$ can be shortened to a single $L_{\infty}$-quasi-isomorphism

$$
F: \mathcal{L} \stackrel{\sim}{\rightarrow} \widetilde{\mathcal{L}} .
$$

Second, for every DG Lie algebra (or possibly an $L_{\infty}$ algebra) $\mathcal{L}$ there exists an $L_{\infty}$-algebra structure on $H^{\bullet}(\mathcal{L})$ such that $H^{\bullet}(\mathcal{L})$ is quasi-isomorphic to $\mathcal{L}$.

Thus if a DG Lie algebra $\mathcal{L}$ is non-formal then this $L_{\infty}$ algebra structure on $H^{\bullet}(\mathcal{L})$ "measures" to what extent $\mathcal{L}$ is far from being formal.

The most pedestrian way to introduce the notion of $L_{\infty}$ algebra is to start with the Chevalley-Eilenberg chain complex $C(\mathcal{L})$ of a DG Lie algebra $\mathcal{L}$.

As a graded vector space the Chevalley-Eilenberg chain complex is the direct sum of all symmetric powers of the desuspension $\mathbf{s}^{-1} \mathcal{L}$ of $\mathcal{L}$

$$
C(\mathcal{L})=\sum_{k=1}^{\infty}\left[\left(\mathbf{s}^{-1} \mathcal{L}\right)^{\otimes k}\right]^{S_{k}} .
$$

To introduce the differential we remark that $C(\mathcal{L})$ is equipped with the following cocommutative comultiplication:

$$
\begin{aligned}
\Delta: C(\mathcal{L}) & \mapsto C(\mathcal{L}) \otimes C(\mathcal{L}), \\
\Delta(v) & =0, \\
\Delta\left(v_{1}, v_{2}, \ldots, v_{n}\right) & =\sum_{k=1}^{n-1} \sum_{\sigma \in \operatorname{Sh}(k, n-k)} \pm\left(v_{\sigma(1)}, \ldots, v_{\sigma(k)}\right) \otimes\left(v_{\sigma(k+1)}, \ldots, v_{\sigma(n)}\right),
\end{aligned}
$$

where $v_{1}, \ldots, v_{n}$ are homogeneous elements of $\mathbf{s}^{-1} \mathcal{L}, \operatorname{Sh}(k, n-k)$ is the set of $(k, n-k)$-shuffles in $S_{n}$, and the signs are determined using the Koszul rule.

To define the boundary operator $Q$ on (2.2) for a DG Lie algebra $\mathcal{L}$ we introduce the natural projection

$$
p: C(\mathcal{L}) \rightarrow \mathbf{s}^{-1} \mathcal{L} .
$$


It is not hard to see that if $Q$ is a coderivation of the coalgebra $C(\mathcal{L})$ in the sense of the equation

$$
\Delta Q=(Q \otimes 1+1 \otimes Q) \Delta
$$

then $Q$ is uniquely determined by its composition $p \circ Q$ with $p$. This statement follows from the fact that $C(\mathcal{L})$ is a cofree ${ }^{2}$ cocommutative coalgebra. The same statement holds for cofree coalgebras of other types. (See Proposition 2.14 in [47].)

Thus we define the coboundary operator $Q$ in terms of the differential $\partial$ and the Lie bracket [, ] by requiring that it is a coderivation of the coalgebra $C(\mathcal{L})$ and by setting

$$
\begin{aligned}
& p \circ Q(v)=-\partial v, \quad p \circ Q\left(v_{1}, v_{2}\right)=(-1)^{\left|v_{1}\right|+1}\left[v_{1}, v_{2}\right], \\
& p \circ Q\left(v_{1}, v_{2}, \ldots, v_{k}\right)=0, \quad k>2,
\end{aligned}
$$

where $v, v_{1}, \ldots, v_{k}$ are homogeneous elements of $\mathcal{L}$.

The equation $Q^{2}=0$ readily follows from the Leibniz rule

$$
\partial\left[v_{1}, v_{2}\right]=\left[\partial v_{1}, v_{2}\right]+(-1)^{\left|v_{1}\right|}\left[v_{1}, \partial v_{2}\right],
$$

and the Jacobi identity:

$$
(-1)^{\left|v_{1}\right|\left|v_{3}\right|}\left[\left[v_{1}, v_{2}\right], v_{3}\right]+\text { c.p. }\{1,2,3\}=0 .
$$

To define a notion of $L_{\infty}$ algebra we simply allow the most general degree 1 coderivation $Q$ of the coalgebra $C(\mathcal{L})(2.2)$ satisfying the equation $Q^{2}=0$. More precisely,

DEFINITION 2. An $L_{\infty}$-algebra structure on a graded vector space $\mathcal{L}$ is a degree 1 coderivation $Q$ of the coalgebra $C(\mathcal{L})(2.2)$ with the comultiplication (2.4) such that

$$
Q^{2}=0 .
$$

Furthermore, an $L_{\infty}$ morphism $F$ from an $L_{\infty}$ algebra $(\mathcal{L}, Q)$ to an $L_{\infty}$ algebra $(\widetilde{\mathcal{L}}, \widetilde{Q})$ is a morphism of the corresponding DG cocommutative coalgebras:

$$
F:(C(\mathcal{L}), Q) \rightarrow(C(\widetilde{\mathcal{L}}), \widetilde{Q}) .
$$

The coderivation $Q$ is uniquely determined by the degree 1 maps

$$
Q_{n}=\left.p \circ Q\right|_{\left(\mathbf{s}^{-1} \mathcal{L}\right)^{\otimes n}}:\left(\mathbf{s}^{-1} \mathcal{L}\right)^{\otimes n} \rightarrow \mathbf{s}^{-1} \widetilde{\mathcal{L}}
$$

and we call them the structure maps of the $L_{\infty}$ algebra $(\mathcal{L}, Q)$.

\footnotetext{
${ }^{2}$ Strictly speaking $C(\mathcal{L})$ is a cofree cocommutative coalgebra without counit.
} 
The equation $Q^{2}=0$ is equivalent to an infinite collection of coherence relations for $Q_{n}$ 's. The first relation says that $Q_{1}^{2}=0$. The second relation is the Leibniz identity for $Q_{1}$ and $Q_{2}$. The third relation says that the binary operation

$$
\left[\gamma_{1}, \gamma_{2}\right]=(-1)^{\left|\gamma_{1}\right|+1} Q_{2}\left(\gamma_{1}, \gamma_{2}\right)
$$

satisfies the Jacobi identity up to homotopy and the corresponding chain homotopy is exactly $Q_{3}$. In particular, the $L_{\infty}$ algebras with zero higher maps $Q_{n}, n>2$, are exactly the DG Lie algebras.

Remark. It is possible to define $\infty$ or homotopy versions for algebras of other types. Although the intrinsic definition of such $\infty$-versions requires the language of operads $[13,47,49,53,71]$, we avoid this language here for the sake of accessibility and try to get by using the vague analogy with the case of $L_{\infty}$ algebras.

By analogy with the coderivations, every morphism (2.6) from the coalgebra $(C(\mathcal{L}), Q)$ to the coalgebra $(C(\widetilde{\mathcal{L}}), \widetilde{Q})$ is uniquely determined by its composition $p \circ F$ with the natural projection

$$
p: C(\widetilde{\mathcal{L}}) \rightarrow \mathbf{s}^{-1} \widetilde{\mathcal{L}}
$$

In other words, an $L_{\infty}$-morphism is not a map from $\mathcal{L}$ to $\widetilde{\mathcal{L}}$ but a collection of (degree zero) maps:

$$
F_{n}=\left.p \circ F\right|_{\left(\mathbf{s}^{-1} \mathcal{L}\right)^{\otimes n}}:\left(\mathbf{s}^{-1} \mathcal{L}\right)^{\otimes n} \mapsto \mathbf{s}^{-1} \widetilde{\mathcal{L}}, \quad n \geq 1
$$

compatible with the action of the symmetric groups $S_{n}$ and satisfying certain equations involving $Q$ and $\widetilde{Q}$.

For this reason we reserve a special arrow $\leadsto$ for $L_{\infty}$-morphisms

$$
F: \mathcal{L} \leadsto \widetilde{\mathcal{L}} .
$$

We call $F_{n}$ (2.8) the structure maps of the $L_{\infty}$ morphism (2.9).

It is not hard to see that the compatibility with $Q$ and $\widetilde{Q}$ implies that $F_{1}$ in (2.8) is a morphism between the cochain complexes $\mathbf{s}^{-1} \mathcal{L}$ and $\mathbf{s}^{-1} \widetilde{\mathcal{L}}$.

DEFINITION 3. An $L_{\infty}$ quasi-isomorphism from $\mathcal{L}$ to $\widetilde{\mathcal{L}}$ is an $L_{\infty}$ morphism (2.9) for which the map $F_{1}: \mathbf{s}^{-1} \mathcal{L} \rightarrow \mathbf{s}^{-1} \widetilde{\mathcal{L}}$ is a quasi-isomorphism of cochain complexes.

Following Hinich we have ${ }^{3}$

THEOREM 1 [54, Lemma 4.2.1]. For every $D G$ Lie algebra $\mathcal{L}$ there exists an $L_{\infty}$ algebra structure $Q^{H}$ on $H^{\bullet}(\mathcal{L})$ such that $\mathcal{L}$ is quasi-isomorphic to the $L_{\infty}$-algebra $\left(H^{\bullet}(\mathcal{L}), Q^{H}\right)$.

\footnotetext{
${ }^{3}$ Hinich [54] proved this statement for $\infty$-versions of algebras over an arbitrary quadratic Koszul operad.
} 
The structure map $Q_{1}^{H}$ is zero and

$$
Q^{H}\left(\gamma_{1}, \gamma_{2}\right)=(-1)^{\left|\gamma_{1}\right|+1}\left[\gamma_{1}, \gamma_{2}\right] \text {, }
$$

where $\gamma_{1}, \gamma_{2} \in H^{\bullet}(\mathcal{L})$ and [, ] is the induced Lie bracket on $H^{\bullet}(\mathcal{L})$.

Thus, even if $\mathcal{L}$ is a non-formal DG Lie algebra, the Lie algebra structure on its cohomology $H^{\bullet}(\mathcal{L})$ can be corrected to an $L_{\infty}$ algebra structure $Q^{H}$ such that the $L_{\infty}$ algebra $\left(H^{\bullet}(\mathcal{L}), Q^{H}\right)$ is quasi-isomorphic to $\mathcal{L}$.

The higher structure maps $Q_{n}^{H}, n>2$ depend on various choices and formality of the DG Lie algebra (or more generally $L_{\infty}$ algebra) $(\mathcal{L}, Q)$ means that one can choose these higher maps to be identically zero.

In the concluding section of this article we give an example of a non-formal DG Lie algebra.

Remark. Using the higher structure maps $Q_{n}^{H}$ one may construct operations which are independent of choices. These operations are known as Massey-Lie products $[6,51,73,78]$ and formality of a DG Lie algebra $\mathcal{L}$ implies that all the Massey-Lie products are zero. A good exposition of Massey-Lie products for the category of DG commutative algebras is given in Section 2 of [6].

\subsection{FROM $L_{\infty}$ ALGEBRAS BACK TO DG LIE ALGEBRAS}

As we see from Definition 2 every $L_{\infty}$ algebra $(\mathcal{L}, Q)$ is defined by the DG cocommutative coalgebra $C(\mathcal{L})$ with the codifferential $Q$. Using this coalgebra we may construct a DG Lie algebra $\mathcal{R}(\mathcal{L}, Q)$ which is quasi-isomorphic to the $L_{\infty}$ algebra $(\mathcal{L}, Q)$. As a graded Lie algebra, $\mathcal{R}(\mathcal{L}, Q)$ is the free Lie algebra generated by $C(\mathcal{L})$

$$
\mathcal{R}(\mathcal{L}, Q)=\operatorname{Lie}(C(\mathcal{L})) .
$$

The differential on $\mathcal{R}(\mathcal{L}, Q)$ consists of two parts. To define the first part we use, in the obvious way, the codifferential $Q$. To define the second part we use the comultiplication $\Delta(2.4)$ on $C(\mathcal{L})$ viewing $(2.10)$ as the dual version of the Harrison chain complex.

The construction of the free resolution $\mathcal{R}(\mathcal{L}, Q)$ for an $L_{\infty}$ algebra $(\mathcal{L}, Q)$ is known in topology as the rectification [14]. We describe this construction in more details for a wider class of algebras in [38] (see Proposition 3 therein).

\subsection{DG IIE ALGEBRAS AND MAURER-CARTAN ELEMENTS}

Given a DG Lie algebra $\mathcal{L}$ with the differential $\partial$ and the Lie bracket [, ] over the field $\mathbb{K}$ we introduce the DG Lie algebra $\mathcal{L}[[\hbar]]$ over ${ }^{4}$ the ring $\mathbb{K}[[\hbar]]$ extending $\partial$ and $[$,$] by \mathbb{K}[[\hbar]]$-linearity.

\footnotetext{
${ }^{4}$ Here $\hbar$ is a formal deformation parameter.
} 
DEFINITION 4. A Maurer-Cartan (or MC) element $\alpha$ of the DG Lie algebra $\mathcal{L}$ is a formal series $\alpha \in \hbar \mathcal{L}^{1}[[\hbar]]$ of degree 1 elements satisfying the equation

$$
\partial \alpha+\frac{1}{2}[\alpha, \alpha]=0 .
$$

MC elements may be formally compared to flat connections.

Let us consider the Lie algebra $\hbar \mathcal{L}^{0}[[\hbar]]$ of formal series of degree zero elements in $\mathcal{L}$. It is easy to see that the Lie algebra $\hbar \mathcal{L}^{0}[[\hbar]]$ is a projective limit of nilpotent Lie algebras:

$$
\hbar \mathcal{L}^{0}[[\hbar]] / \hbar^{N} \mathcal{L}^{0}[[\hbar]], \quad N>1 .
$$

Therefore it can be exponentiated to the group

$$
\mathfrak{G}=\exp \left(\hbar \mathcal{L}^{0}[[\hbar]]\right) .
$$

This group acts on the MC elements of $\mathcal{L}$ according to the formula:

$$
\exp (\xi) \alpha=\exp ([, \xi]) \alpha+f([, \xi]) \partial \xi,
$$

where $f$ is the power series of the function

$$
f(x)=\frac{e^{x}-1}{x}
$$

at the point $x=0$. Two MC elements connected by the action of the group $\mathfrak{G}$ may be thought of as equivalent flat connections.

In this way we get the Goldman-Millson groupoid [50] $\mathrm{MC}(\mathcal{L})$ which captures the formal one-parameter deformation theory associated to the DG Lie algebra $\mathcal{L}$. Objects of this groupoid are MC elements of $\mathcal{L}$ and morphisms between two MC elements $\alpha_{1}$ and $\alpha_{2}$ are elements of the group $\mathfrak{G}$ (2.12) which transform $\alpha_{1}$ to $\alpha_{2}$.

We denote by $\pi_{0}(\operatorname{MC}(\mathcal{L}))$ the set of isomorphism classes of the GoldmanMillson groupoid $\mathrm{MC}(\mathcal{L})$.

Every morphism $\mu: \mathcal{L} \rightarrow \widetilde{\mathcal{L}}$ of DG Lie algebras gives us an obvious functor

$$
\mu_{*}: \operatorname{MC}(\mathcal{L}) \rightarrow \operatorname{MC}(\widetilde{\mathcal{L}})
$$

from the groupoid $\operatorname{MC}(\mathcal{L})$ to the groupoid $\operatorname{MC}(\widetilde{\mathcal{L}})$.

According to $[46,50,77]$ we have the following theorem

THEOREM 2. If $\mu: \mathcal{L} \rightarrow \widetilde{\mathcal{L}}$ is a quasi-isomorphism of $D G$ Lie algebras then $\mu_{*}$ induces a bijection between $\pi_{0}(\mathrm{MC}(\mathcal{L}))$ and $\pi_{0}(\mathrm{MC}(\widetilde{\mathcal{L}}))$.

Theorem 2 is an immediate corollary of Proposition 4.9 in Getzler's paper [46]. According to Goldman and Millson [50] and Schlessinger and Stasheff [77] every quasi-isomorphism $\mu$ from $\mathcal{L}$ to $\widetilde{\mathcal{L}}$ induces an equivalence of groupoids $\operatorname{MC}(\mathcal{L})$ 
and $\operatorname{MC}(\widetilde{\mathcal{L}})$ provided the DG Lie algebras $\mathcal{L}$ and $\widetilde{\mathcal{L}}$ are concentrated in nonnegative degrees.

It turns out that if $\mathcal{L}$ has elements in negative degrees then $\operatorname{MC}(\mathcal{L})$ can be upgraded to a higher groupoid [45]. For $L_{\infty}$ algebras this idea was thoroughly developed by Getzler in [46]. Then these results of Getzler were generalized by Henriques [52].

\subsection{TWISTING BY A MC ELEMENT}

Given a MC element $\alpha \in \hbar \mathcal{L}[[\hbar]]$ of a DG Lie algebra $\mathcal{L}$ we may modify the DG Lie algebra structure on $\mathcal{L}[[\hbar]]$ by switching to the new differential

$$
\partial+[\alpha,]
$$

It is the MC equation (2.11) which implies the identity $(\partial+[\alpha,])^{2}=0$.

We denote the DG Lie algebra $\mathcal{L}[[\hbar]]$ with the differential (2.15) and the original Lie bracket by $\mathcal{L}^{\alpha}$

$$
\mathcal{L}^{\alpha}=(\mathcal{L}[[\hbar]], \partial+[\alpha,],[,]) .
$$

Following Quillen [75] we call this procedure of modifying the DG Lie algebra twisting.

It is obvious that every morphism $\mu: \mathcal{L} \rightarrow \widetilde{\mathcal{L}}$ of DG Lie algebras extends by $\mathbb{K}[[\hbar]]$-linearity to a morphism from $\mathcal{L}^{\alpha}$ to $\widetilde{\mathcal{L}}^{\mu(\alpha)}$. We denote this morphism by $\mu^{\alpha}$.

We claim that

PROPOSITION 1 [34, Proposition 1]. If $\mu: \mathcal{L} \rightarrow \widetilde{\mathcal{L}}$ is a quasi-isomorphism between $D G$ Lie algebras then so is the morphism

$$
\mu^{\alpha}: \mathcal{L}^{\alpha} \rightarrow \widetilde{\mathcal{L}}^{\mu(\alpha)} .
$$

For $L_{\infty}$ algebras, the twisting procedure was described in [46].

\section{Algebraic Structures on the Hochschild Complexes}

Let us introduce the algebras we are interested in.

First, we recall from [44] that

DEFINITION 5. A graded vector space $V$ is a Gerstenhaber algebra if it is equipped with a graded commutative and associative product $\wedge$ of degree 0 and a graded Lie bracket [, ] of degree -1 . These operations have to be compatible in the sense of the following Leibniz rule

$$
\left[\gamma, \gamma_{1} \wedge \gamma_{2}\right]=\left[\gamma, \gamma_{1}\right] \wedge \gamma_{2}+(-1)^{\left|\gamma_{1}\right|(|\gamma|+1)} \gamma_{1} \wedge\left[\gamma, \gamma_{2}\right]
$$


Second, we recall from [29] that

DEFINITION 6. A precalculus is a pair of a Gerstenhaber algebra $(V, \wedge,[]$,$) and$ a graded vector space $W$ together with

- a module structure $i_{\bullet}: V \otimes W \mapsto W$ of the graded commutative algebra $V$ on $W$, - an action $l_{\bullet}: \mathbf{s}^{-1} V \otimes W \mapsto W$ of the Lie algebra $\mathbf{s}^{-1} V$ on $W$ which is compatible with $i_{\bullet}$ in the sense of the following equations

$$
i_{a} l_{b}-(-1)^{|a|(|b|+1)} l_{b} i_{a}=i_{[a, b]}, \quad l_{a \wedge b}=l_{a} i_{b}+(-1)^{|a|} i_{a} l_{b} .
$$

Furthermore,

DEFINITION 7. A calculus is a precalculus $\left(V, W,[],, \wedge, i_{\bullet}, l_{\bullet}\right)$ with a degree -1 unary operation $\delta$ on $W$ such that

$$
\delta i_{a}-(-1)^{|a|} i_{a} \delta=l_{a},
$$

$\operatorname{and}^{5} \delta^{2}=0$.

The simplest examples of these algebraic structures come from geometry. More precisely, if $M$ is a smooth real manifold then the graded vector space $T_{\text {poly }}^{\bullet}(M)$ of polyvector fields on $M$ is a Gerstenhaber algebra. The commutative product is simply the exterior product $\wedge$ and the Lie bracket is the Schouten-Nijenhuis bracket

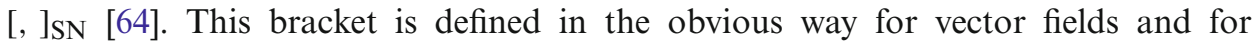
functions:

$$
\begin{aligned}
& {\left[v_{1}, v_{2}\right]_{\mathrm{SN}}=\left[v_{1}, v_{2}\right], \quad[v, a]_{\mathrm{SN}}=v(a), \quad[a, b]_{\mathrm{SN}}=0,} \\
& v, v_{1}, v_{2} \in T_{\text {poly }}^{1}(M), \quad a, b \in T_{\text {poly }}^{0}(M)=C^{\infty}(M) .
\end{aligned}
$$

Then it is extended by the Leibniz rule (3.1) to all polyvector fields.

Adding to polyvector fields the graded vector space $\Omega^{-\bullet}(M)$ of exterior forms with the reversed grading

$$
\left(T_{\text {poly }}^{\bullet}(M), \Omega^{-\bullet}(M)\right)
$$

we get a calculus algebra. The module structure on $\Omega^{-\bullet}(M)$ over the commutative algebra $\left(T_{\text {poly }}^{\bullet}(M), \wedge\right)$ is defined by the contraction

$$
i: T_{\text {poly }}^{\bullet}(M) \otimes \Omega^{-\bullet}(M) \rightarrow \Omega^{-\bullet}(M),
$$

\footnotetext{
${ }^{5}$ Although $\delta^{2}=0$, the operation $\delta$ is not considered as a part of the differential on $W$.
} 
the unary operation $\delta$ is the de Rham differential $d$ and the Lie algebra module structure on $\Omega^{-\bullet}(M)$ over $\left.\left(T_{\text {poly }}^{\bullet+1}(M) \text {, [, ] }\right]_{\mathrm{SN}}\right)$ is given by the Lie derivative

$$
\begin{aligned}
& l: T_{\text {poly }}^{\bullet+1}(M) \otimes \Omega^{-\bullet}(M) \rightarrow \Omega^{-\bullet}(M), \\
& l_{\gamma}=d i_{\gamma}-(-1)^{|\gamma|} i_{\gamma} d .
\end{aligned}
$$

Another example of calculus algebra comes from non-commutative geometry. To give this example we start with an arbitrary unital associative algebra $A$.

For every bimodule $U$ over the algebra $A$ we introduce the (normalized) Hochschild cochain complex

$$
C^{\bullet}(A, U)=\operatorname{Hom}\left((A / \mathbb{K} 1)^{\otimes \bullet}, U\right)
$$

and the (normalized) Hochschild chain complex

$$
C_{\bullet}(A, U)=U \otimes(A / \mathbb{K} 1)^{\otimes \bullet}
$$

of $A$ with coefficients in $U$. It should be remarked that, in this paper, we only use normalized versions of Hochschild complexes.

We reserve the same notation $\partial^{\text {Hoch }}$ both for the Hochschild coboundary operator

$$
\begin{aligned}
& \left(\partial^{\text {Hoch }} P\right)\left(a_{0}, a_{1}, \ldots, a_{k}\right)=(-1)^{k+1}\left(a_{0} P\left(a_{1}, \ldots, a_{k}\right)-P\left(a_{0} a_{1}, \ldots, a_{k}\right)+\right. \\
& \quad+P\left(a_{0}, a_{1} a_{2}, a_{3}, \ldots, a_{k}\right)-\cdots+(-1)^{k} P\left(a_{0}, \ldots, a_{k-2}, a_{k-1} a_{k}\right)+ \\
& \left.\quad+(-1)^{k+1} P\left(a_{0}, \ldots, a_{k-2}, a_{k-1}\right) a_{k}\right)
\end{aligned}
$$

on $C^{\bullet}(A, U)$ and for Hochschild boundary operator

$$
\begin{aligned}
& \partial^{\text {Hoch }}\left(u, a_{1}, \ldots, a_{m}\right)=\left(u a_{1}, a_{2}, \ldots, a_{m}\right)-\left(u, a_{1} a_{2}, a_{3}, \ldots, a_{m}\right)+\cdots+ \\
& +(-1)^{m-1}\left(u, a_{1}, \ldots, a_{m-2}, a_{m-1} a_{m}\right)+(-1)^{m}\left(a_{m} u, a_{1}, a_{2}, \ldots, a_{m-1}\right), \\
& \quad a_{i} \in A, \quad u \in U
\end{aligned}
$$

on $C_{\bullet}(A, U)$.

We reserve the notation $H H^{\bullet}(A, U)$ (resp. $H H_{\bullet}(A, U)$ ) for the Hochschild cohomology (resp. homology) groups of $A$ with coefficients in $U$.

In the case $U=A$ we simplify the notation for the Hochschild complexes and for the (co)homology groups:

$$
\begin{aligned}
C^{\bullet}(A) & =C^{\bullet}(A, A), \\
C_{\bullet}(A) & =C_{-\bullet}(A, A), \\
H H^{\bullet}(A) & =H H^{\bullet}(A, A), \quad H H_{\bullet}(A)=H H_{-\bullet}(A, A) .
\end{aligned}
$$

For our purposes, we use the reversed grading on the Hochschild chains of $A$ with coefficients in $A$.

Here are the five algebraic operations on the complexes $C^{\bullet}(A)$ and $C_{\bullet}(A)$ which play an important role: 
- the cup-product $U$

$$
\begin{aligned}
& P_{1} \cup P_{2}\left(a_{1}, a_{2}, \ldots, a_{k_{1}+k_{2}}\right)=P_{1}\left(a_{1}, \ldots, a_{k_{1}}\right) P_{2}\left(a_{k_{1}+1}, \ldots, a_{k_{1}+k_{2}}\right), \\
& \quad P_{i} \in C^{k_{i}}(A),
\end{aligned}
$$

- the Gerstenhaber bracket $[,]_{G}$

$$
\begin{aligned}
& {\left[Q_{1}, \underset{Q_{2}}{Q_{G}}\right]_{G}=} \\
& =\sum_{i=0}^{k_{1}}(-1)^{i k_{2}} Q_{1}\left(a_{0}, \ldots, Q_{2}\left(a_{i}, \ldots, a_{i+k_{2}}\right), \ldots, a_{k_{1}+k_{2}}\right)-(-1)^{k_{1} k_{2}}(1 \leftrightarrow 2), \\
& \quad Q_{i} \in C^{k_{i}+1}(A),
\end{aligned}
$$

- the contraction $I_{P}$ of a Hochschild cochain $P \in C^{k}(A)$ with Hochschild chains

$$
I_{P}\left(a_{0}, a_{1}, \ldots, a_{m}\right)=\left\{\begin{array}{l}
(-1)^{k m}\left(a_{0} P\left(a_{1}, \ldots, a_{k}\right), a_{k+1}, \ldots, a_{m}\right), \quad \text { if } m \geq k, \\
0, \quad \text { otherwise, }
\end{array}\right.
$$

- the Lie derivative of Hochschild chains along a Hochschild cochain $Q \in C^{k+1}(A)$

$$
\begin{aligned}
& L_{Q}\left(a_{0}, a_{1}, \ldots, a_{m}\right)=\sum_{i=0}^{m-k}(-1)^{k i}\left(a_{0}, \ldots, Q\left(a_{i}, \ldots, a_{i+k}\right), \ldots, a_{m}\right)+ \\
& \quad+\sum_{j=m-k}^{m-1}(-1)^{m(j+1)}\left(Q\left(a_{j+1}, \ldots, a_{m}, a_{0}, \ldots, a_{k+j-m}\right), a_{k+j+1-m}, \ldots, a_{j}\right),
\end{aligned}
$$

- and Connes' operator $B: C_{\bullet}(A) \rightarrow C_{\bullet-1}(A)$

$$
\begin{aligned}
B\left(a_{0}, \ldots, a_{m}\right)= & \sum_{i=0}^{m}\left((-1)^{m i}\left(1, a_{i}, \ldots, a_{m}, a_{0}, \ldots, a_{i-1}\right)+\right. \\
& \left.+(-1)^{m i}\left(a_{i}, 1, a_{i+1}, \ldots, a_{m}, a_{0}, \ldots, a_{i-1}\right)\right) .
\end{aligned}
$$

All these operations are compatible with the differential $\partial^{\text {Hoch }}$ (3.8), (3.9). Therefore they induce the corresponding operations on the level of cohomology.

There are several identities involving the operations $[,]_{G}, L$, and $B$.

First, the Gerstenhaber bracket $[,]_{G}$ is a Lie bracket on $C^{\bullet+1}(A)$ and hence $C^{\bullet+1}(A)$ is a DG Lie algebra. The operation $L$ (3.16) gives us an action of the DG Lie algebra $C^{\bullet+1}(A)$ on Hochschild chains. In other words,

$$
L_{Q_{1}} L_{Q_{2}}-(-1)^{\left(\left|Q_{1}\right|+1\right)\left(\left|Q_{2}\right|+1\right)} L_{Q_{2}} L_{Q_{1}}=L_{\left[Q_{1}, Q_{2}\right]_{G}}
$$

and hence $C_{\bullet}(A)$ is a DG Lie algebra module over the DG Lie algebra $C^{\bullet+1}(A)$. 
The Connes cyclic operator $B$ (3.17) is used in the definitions of different variants of cyclic chain complex $[29,68]$. All these variants have the form ${ }^{6}$

$$
C C_{\bullet}^{\mathcal{W}}(A)=\left(C_{\bullet}(A)[[u]] \otimes_{\mathbb{K}[u]} \mathcal{W}, \partial^{\text {Hoch }}+u B\right),
$$

where $u$ is an auxiliary variable of degree 2 and $\mathcal{W}$ is a $\mathbb{K}[u]$-module.

We are interested in two particular cases:

- if $\mathcal{W}=\mathbb{K}[u]$ then $C C_{\bullet}^{\mathcal{W}}(A)$ is called the negative cyclic complex

$$
C C_{\bullet}^{-}(A)=\left(C_{\bullet}(A)[[u]], \partial^{\text {Hoch }}+u B\right),
$$

- if $\mathcal{W}=\mathbb{K}$ with $u$ acting by zero then $C C_{\bullet}^{\mathcal{W}}(A)$ is nothing but the Hochschild chain complex (3.11) of $A$.

Since the Connes cyclic operator $B$ (3.17) is compatible with the "Lie derivative" $L$ (3.16) in the sense of the equation

$$
B L_{P}-(-1)^{|P|+1} L_{P} B=0, \quad P \in C^{\bullet}(A)
$$

any variant of the cyclic chain complex (3.19) is a DG module over the DG Lie algebra $C^{\bullet+1}(A)$.

Unfortunately, the cup-product $\cup$ and the Gerstenhaber bracket $[,]_{G}$ do not satisfy the Leibniz rule. So $\cup$ and $[,]_{G}$ do not give us a Gerstenhaber algebra structure on $C^{\bullet}(A)$. Similarly, the operations $\cup,[,]_{G}, I, L, B$ do not give us a calculus algebra on the pair $\left(C^{\bullet}(A), C_{\bullet}(A)\right)$. However, the required identities hold on the level of cohomology and we have

PROPOSITION 2 [44]. The cup-product (3.13) and the bracket (3.14) induce on $H H^{\bullet}(A)$ a structure of a Gerstenhaber algebra.

and

PROPOSITION 3 [30]. The operations (3.13), (3.14), (3.15), (3.16), and (3.17) induce on the pair

$$
\left(H H^{\bullet}(A), H H_{\bullet}(A)\right)
$$

a structure of a calculus.

Let us also recall the Van den Bergh duality theorem:

THEOREM 3 [88]. If $A$ is a finitely generated bimodule coherent ${ }^{7}$ algebra of finite Hochschild dimension $d$,

$$
H H^{m}(A, A \otimes A)=0
$$

${ }^{6}$ This notation is due to E. Getzler.

${ }^{7} \mathrm{An}$ algebra $A$ is called bimodule coherent if every map between finite rank free $A$-bimodules has a finitely generated kernel (see Definition 3.5.1 in [48]). 
for all $m \neq d$, and if

$$
V_{A}:=H H^{d}(A, A \otimes A)
$$

is an invertible ${ }^{8}$ A-bimodule, then for every A-bimodule $U$,

$$
H H^{\bullet}(A, U) \cong H H_{d-\bullet}\left(A, V_{A} \otimes_{A} U\right) .
$$

In Equations (3.21) and (3.22) $A \otimes A$ is considered as a bimodule over $A$ with respect to the external $A$-bimodule structure. It is the internal $A$-bimodule structure which equips all the cohomology groups $H H^{\bullet}(A, A \otimes A)$ with a structure of A-bimodule.

We refer to $V_{A}$ in (3.22) as the Van den Bergh dualizing module of $A$.

\section{Formality Theorems}

The famous Kontsevich's formality theorem can beformulated as

THEOREM 4 [58]. Let $M$ be a smooth real manifold and $A=C^{\infty}(M)$ be the algebra of smooth functions on $M$. Then the DG Lie algebra $\left(C^{\bullet+1}(A), \partial^{\text {Hoch }},[,]_{G}\right)$ of Hochschild cochains is quasi-isomorphic to the graded Lie algebra $\left(T_{\text {poly }}^{\bullet+1}(M),[,]_{\mathrm{SN}}\right)$ of polyvector fields.

Remark. Since $A=C^{\infty}(M)$ is a topological algebra the definition of (normalized) Hochschild cochains for $A$ requires some precaution. By (normalized) Hochschild cochains of the algebra $A=C^{\infty}(M)$ we mean polydifferential operators $P$ on $M$ satisfying the property

$$
P(\ldots, 1, \ldots)=0 .
$$

To prove Theorem 4, Kontsevich gave [58] an explicit construction of an $L_{\infty}$ quasi-isomorphism

$$
\mathcal{K}: T_{\text {poly }}^{\bullet+1}\left(\mathbb{R}^{d}\right) \sim C^{\bullet+1}\left(C^{\infty}\left(\mathbb{R}^{d}\right)\right)
$$

from the graded Lie algebra $T_{\text {poly }}^{\bullet+1}\left(\mathbb{R}^{d}\right)$ of polyvector fields to the DG Lie algebra $C^{\bullet+1}\left(C^{\infty}\left(\mathbb{R}^{d}\right)\right)$ of polydifferential operators on $\mathbb{R}^{d}$. This construction involves very interesting integrals over compactified configuration spaces of points on the upper half-plane. Tedious questions about choices of signs were thoroughly addressed in [5].

In order to extend this result to an arbitrary smooth manifold Kontsevich used what is called the Gelfand-Fuchs trick [42] or the formal geometry [43] in the sense of Gelfand and Kazhdan.

\footnotetext{
${ }^{8} \mathrm{An} A$-bimodule $V$ is called invertible if there is an $A$-bimodule $\widetilde{V}$ such that $V \otimes_{A} \widetilde{V} \cong A$.
} 
This step of globalization was discussed later in more details by Cattaneo et al. [25], by Kontsevich in Appendix 3 in [60], by the first author in [32], by Yekutieli in [96] and by Van den Bergh in [89].

To describe the first immediate corollary of Theorem 4 we recall from $[7,8,10,11]$ that a star-product on the manifold $M$ is an $\mathbb{R}[[\hbar]]$-linear associative product on $C^{\infty}(M)[[\hbar]]$ of the form

$$
a * b=a b+\sum_{k=1}^{\infty} \hbar^{k} \Pi_{k}(a, b), \quad a, b \in C^{\infty}(M)[[\hbar]],
$$

where $\Pi_{k}$ are bidifferential operators.

Since $a * b=a b$ mod $\hbar$ the star-product (4.2) should be viewed as an associative and not necessarily commutative formal deformation of the ordinary product of functions on $M$.

Two star-products $*$ and $\tilde{*}$ are called equivalent if there exists a formal series of differential operators

$$
T=I d+\hbar T_{1}+\hbar^{2} T_{2}+\cdots,
$$

which starts from the identity and intertwines the star-products $*$ and $\tilde{*}$ :

$$
T(a * b)=T(a) \tilde{*} T(b) .
$$

It is easy to see that the associativity property of the star-product (4.2) is equivalent to the MC equation

$$
\partial^{\text {Hoch }} \Pi+\frac{1}{2}[\Pi, \Pi]_{G}=0
$$

for the element

$$
\Pi=\sum_{k=1}^{\infty} \hbar^{k} \Pi_{k} .
$$

Thus MC elements of the DG Lie algebra $C^{\bullet+1}\left(C^{\infty}(M)\right)$ are exactly the starproducts on $M$.

Furthermore, it is not hard to identify the intertwiners between star-products with morphisms of the Goldman-Millson groupoid $\mathrm{MC}\left(C^{\bullet+1}\left(C^{\infty}(M)\right)\right)$.

For the graded Lie algebra $T_{\text {poly }}^{\bullet+1}(M)$ of polyvector fields, the MC elements are the formal Poisson structures. These are formal power series of bivectors

$$
\pi=\sum_{k=1}^{\infty} \hbar^{k} \pi_{k}, \quad \pi_{k} \in T_{\text {poly }}^{2}(M)
$$

satisfying the Jacobi relation

$$
[\pi, \pi]_{\mathrm{SN}}=0 .
$$


We say that two formal Poisson structures $\pi$ and $\tilde{\pi}$ are equivalent if they are connected by the adjoint action of the group

$$
\exp \left(\hbar T_{\text {poly }}^{1}(M)[[\hbar]]\right) \text {. }
$$

Thus combining Theorems 2 and 4 we get the following corollary

COROLLARY 1. Equivalence classes of star products are in bijection with the equivalence classes of formal Poisson structures.

Given a star-product $*$ on $M$, we call the corresponding equivalence class of formal Poisson structures the Kontsevich class of the star-product. Notice that the action of the group (4.8) does not change the first term $\pi_{1}$ of the series (4.6). Thus $\pi_{1}$ does not depend on the choice of the representative (4.6) of the Kontsevich class. We refer to $\pi_{1}$ as the Poisson bivector corresponding to the star-product $*$.

Twisting the DG Lie algebra $C^{\bullet+1}\left(C^{\infty}(M)\right)$ by the MC element $\Pi$ (4.5) corresponding to the star-product $*$ (4.2) we get the DG Lie algebra $C^{\bullet+1}\left(A_{\hbar}\right)$ of Hochschild cochains for the deformation quantization algebra $A_{\hbar}=\left(C^{\infty}(M)[[\hbar]], *\right)$.

Twisting the graded Lie algebra of polyvector fields $T_{\text {poly }}^{\bullet+1}(M)$ by the MC element $\pi$ (4.6) we get the Poisson cochain complex [67]

$$
\left(T_{\text {poly }}^{\bullet+1}(M)[[\hbar]],[\pi,]_{\mathrm{SN}}\right)
$$

of $\pi$. Omitting the shift we refer to the cohomology of the complex (4.9) as the Poisson cohomology $H P^{\bullet}(M, \pi)$ of the formal Poisson structure $\pi$

$$
H P^{\bullet}(M, \pi):=H^{\bullet}\left(T^{c} \bullet \text { poly }(M)[[\hbar]],[\pi,]_{\mathrm{SN}}\right) .
$$

Thus, combining Proposition 1 with Theorems 2 and 4, we get the following corollary

COROLLARY 2. If $*$ is a star-product whose Kontsevich class is represented by the formal Poisson structure $\pi$ then the Hochschild cohomology of the deformation quantization algebra $A_{\hbar}=\left(C^{\infty}(M)[[\hbar]], *\right)$ is isomorphic to the Poisson cohomology $H P^{\bullet}(M, \pi)$ of the formal Poisson structure $\pi$.

\subsection{ALTERNATIVE APPROACH TO THEOREM 4}

As we mentioned above, the operations $\cup$ (3.13) and []$_{G}$ (3.14) do not equip the Hochschild cochain complex $C^{\bullet}(A)$ with a Gerstenhaber algebra structure.

However, using the solution of Deligne's Hochschild cohomological conjecture $[12,62,74,84,92]$ one can show that the operations $U$ and $[,]_{G}$ can be upgraded to an $\infty$-Gerstenhaber algebra whose multiplications are expressed in terms of the cup-product $U$ and insertions of cochains into a cochain. 
This $\infty$-Gerstenhaber structure depends on the choice of a Drinfeld associator [40]. At this moment it is not known whether the homotopy type of this $\infty$-Gerstenhaber structure depends on this choice. However,

THEOREM 5 [82, Theorem 2.1]. The $L_{\infty}$ algebra part of the $\infty$-Gerstenhaber structure on Hochschild cochains coincides with the DG Lie algebra structure given by the Hochschild differential and the Gerstenhaber bracket (3.14).

In 1998 the second author proposed a completely different proof [82] of Theorem 4 in the case when $A$ is the polynomial algebra over an arbitrary field $\mathbb{K}$ of characteristic zero. We would like to refer the reader to Hinich's excellent exposition [54] of this approach.

In [82] it was shown that the Gerstenhaber algebra $T_{\text {poly }}^{\bullet}\left(\mathbb{K}^{d}\right)$ of polyvector fields on the affine space $\mathbb{K}^{d}$ is intrinsically formal. In other words, there is no room for cohomological obstructions to the formality of any $\infty$-Gerstenhaber algebra whose cohomology is the Gerstenhaber algebra $T_{\text {poly }}^{\bullet}\left(\mathbb{K}^{d}\right)$.

Therefore, the Hochschild cochain complex $C^{\bullet}\left(\mathbb{K}\left[x_{1}, \ldots, x_{d}\right]\right)$ with the $\infty$-Gerstenhaber structure coming from the solution of Deligne's conjecture is formal.

Combining this observation with Theorem 5 we immediately deduce Theorem 4 . At this moment there is a more explicit proof of the formality for the $\infty$-Gerstenhaber structure for a wider class of algebras. More precisely,

THEOREM 6 [38]. For every regular commutative algebra A over a field $\mathbb{K}$ of characteristic zero, the $\infty$-Gerstenhaber algebra $C^{\bullet}(A)$ of Hochschild cochains is formal.

An analogous statement in the Lie algebroid setting was proved by Calaque and Van den Bergh [20].

\subsection{FORMALITY THEOREMS FOR HOCHSCHILD AND CYCLIC CHAINS}

Hochschild chains (3.11) enter this picture in a very natural way.

THEOREM 7. Let $M$ be a smooth real manifold and $A=C^{\infty}(M)$ be the algebra of smooth functions on $M$. Then the pair "DG Lie algebra $C^{\bullet+1}(A)$ and its $D G$ module $C .(A)$ " is quasi-isomorphic to the pair "graded Lie algebra $T_{\text {poly }}^{\bullet+1}(M)$ and its module $\Omega^{-\bullet}(M)$ ".

This statement was formulated as a conjecture by the third author in [87]. It was proved in [79] by Shoikhet for the case $M=\mathbb{R}^{d}$. The step of globalization was performed in the thesis of the first author [33,34].

As well as Theorem 4, Theorem 7 requires a technical amendment. More precisely, the space $C_{k}(A)$ of degree $k$ (normalized) Hochschild chains for the algebra 
$A=C^{\infty}(M)$ should be replaced by the space of (normalized) $\infty$-jets near the main diagonal of the product $M^{\times k}$.

In [87] the third author also conjectured the formality of the cyclic complexes (3.19) as DG Lie algebra modules over $C^{\bullet+1}(A)$. In [85] the second author and the third author proposed a plan for proving this cyclic conjecture.

However, in [94] Willwacher showed elegantly that Shoikhet's $L_{\infty}$ quasi-isomorphism [79] is compatible with the Connes cyclic operator (3.17). This observation readily settled in the positive Tsygan's cyclic formality conjecture:

THEOREM 8 [94]. Let $M$ be a smooth real manifold and $A=C^{\infty}(M)$ be the algebra of smooth functions on $M$. If $\mathcal{W}$ is an $\mathbb{R}[u]$-module of finite projective dimension, then the pair " $D G$ Lie algebra $C^{\bullet+1}(A)$ and its $D G$ module $C C_{\bullet}^{\mathcal{W}}(A)$ " is quasi-isomorphic to the pair "graded Lie algebra $T_{\text {poly }}^{\bullet+1}(M)$ and its $D G$ module $\left(\Omega^{-\bullet}(M)[[u]] \otimes_{\mathbb{R}[u]} \mathcal{W}, u d\right)$.

If we set $\mathcal{W}=\mathbb{R}$ with $u$ acting by zero then $C C_{\bullet}^{\mathcal{W}}(A)$ becomes the Hochschild chain complex and the DG module

$$
\left(\Omega^{-\bullet}(M)[[u]] \otimes_{\mathbb{R}[u]} \mathcal{W}, u d\right)
$$

becomes the module $\Omega^{-\bullet}(M)$ with the zero differential. Thus Theorem 7 is a corollary of Theorem 8 .

Given a Lie algebra $V$ and a $V$-module $W$, we can form the semi-direct product $V \oplus W$ in which $W$ is an Abelian Lie algebra. It is clear that pairs "Lie algebra $V$ and its module $W$ " can be identified with such semi-direct products.

Using this idea one can generalize the twisting procedure we described in Section 2.4 to DG Lie algebra modules.

Thus, if $*$ is a star-product on $M$ then twisting the DG Lie algebra module of negative cyclic chains (3.20) of the algebra $A=C^{\infty}(M)$ by the corresponding MC element $\Pi$ (4.5) we get the negative cyclic complex

$$
C C_{\bullet}^{-}\left(A_{\hbar}\right)
$$

for the deformation quantization algebra $A_{\hbar}=\left(C^{\infty}(M)[[\hbar]], *\right)$.

Let $\pi$ be a formal Poisson structure (4.6). Regarding $\pi$ as a MC element of the graded Lie algebra $T_{\text {poly }}^{\bullet}(M)$ and twisting its DG Lie algebra module

$$
\left(\Omega^{-\bullet}(M)[[u]], u d\right)
$$

we get the DG Lie algebra module

$$
\left(\Omega^{-\bullet}(M)[[u]][[\hbar]], l_{\pi}+u d\right)
$$

over the DG Lie algebra $\left(T_{\text {poly }}^{\bullet}(M)[[\hbar]],[\pi,]_{\mathrm{SN}}\right)$ with the Lichnerowicz differential $[\pi,]_{\mathrm{SN}}$. 
Generalizing Proposition 1 to DG Lie algebra modules in the obvious way, we get the following corollary of Theorem 8

COROLLARY 3. If $*$ is a star-product on $M$ whose Kontsevich class is represented by the formal Poisson structure $\pi$, then the complex (4.11) computes the negative cyclic homology

$$
H C_{\bullet}^{-}\left(A_{\hbar}\right)=H^{\bullet}\left(C C_{\bullet}^{-}\left(A_{\hbar}\right)\right)
$$

of the deformation quantization algebra $A_{\hbar}=\left(C^{\infty}(M)[[\hbar]], *\right)$.

Remark. Applying similar arguments to the Hochschild chain complex and to the exterior forms we get an isomorphism between Hochschild homology of $A_{\hbar}$ and the Poisson homology $[16,64]$ of $\pi$.

\subsection{FORMALITY OF THE $\infty$-CALCULUS ALGEBRA $\left(C^{\bullet}(A), C \bullet(A)\right)$}

To include the operations $\cup$ (3.13), $[,]_{G}$ (3.14), $I$ (3.15), $L$ (3.16), and $B$ (3.17) on the pair

$$
\left(C^{\bullet}(A), C_{\bullet}(A)\right)
$$

into the picture we need to find a correct algebraic structure on (4.12).

As we already mentioned above the operations $\cup(3.13),[,]_{G}(3.14), I(3.15), L$ (3.16), and $B$ (3.17) satisfy the identities of the calculus algebra only up to homotopy. So a calculus algebra is not a correct algebraic structure for this situation.

Luckily the Kontsevich-Soibelman solution [63] of the chain version of Deligne's conjecture implies that the operations $\cup,[,]_{G}, I, L$, and $B$ can be upgraded to an $\infty$-calculus algebra whose multiplications are expressed in terms of the cupproduct $\cup$, insertions of cochains into a cochain, and insertions of components of a chain into cochains which respect the cyclic order on these components.

In [39] we show that

THEOREM 9 [39, Corollary 4]. For every smooth real manifold $M$ the $\infty$-calculus algebra

$$
\left(C^{\bullet}\left(C^{\infty}(M)\right), C_{\bullet}\left(C^{\infty}(M)\right)\right)
$$

is quasi-isomorphic to the calculus algebra

$$
\left(T_{\bullet}^{\text {poly }}(M), \Omega^{-\bullet}(M)\right)
$$

of polyvector fields and exterior forms on $M$.

We prove this theorem using our construction from [38] for the $\infty$-Gerstenhaber algebra on $C^{\bullet}\left(C^{\infty}(M)\right)$ and the Morita equivalence between the algebra of differential operators on exterior forms and the algebra of differential operators on 
functions. Unlike in [38], we did not produce an explicit sequence of quasi-isomorphisms connecting (4.13) and (4.14). So Theorem 9 has the status of an existence theorem.

Theorem 4 from [39] implies that in the algebra structure on (4.12), operations $[,]_{G}, L$ and $B$ do not have higher homotopy corrections inside the $\infty$-calculus structure. Thus the cyclic formality theorem of Willwacher (Theorem 8) is a corollary of Theorem 9.

We should remark that Kontsevich's $L_{\infty}$ quasi-isomorphism $\mathcal{K}(4.1)$ has a subtle compatibility property with the cup-product. To formulate this property we let $\pi$ be a formal Poisson structure (4.6) on $\mathbb{R}^{d}$ and $A_{\hbar}=\left(C^{\infty}\left(\mathbb{R}^{d}\right)[[\hbar]], *\right)$ be a deformation quantization algebra whose Kontsevich class is represented by $\pi$. Twisting Kontsevich's $L_{\infty}$ quasi-isomorphism $\mathcal{K}(4.1)$ by $\pi$ we get the $L_{\infty}$ quasi-isomorphism

$$
\mathcal{K}^{\pi}:\left(T_{\text {poly }}^{\bullet+1}\left(\mathbb{R}^{d}\right)[[\hbar]],[\pi,]_{\mathrm{SN}}\right) \leadsto C^{\bullet+1}\left(A_{\hbar}\right),
$$

which, in turn, induces an isomorphism from the Poisson cohomology

$$
H P^{\bullet}\left(\mathbb{R}^{d}, \pi\right):=H^{\bullet}\left(T_{\text {poly }}^{\bullet}\left(\mathbb{R}^{d}\right)[[\hbar]],[\pi,]_{\mathrm{SN}}\right) .
$$

to the Hochschild cohomology

$$
H H^{\bullet}\left(A_{\hbar}\right)
$$

of $A_{\hbar}$.

The exterior product $\wedge$ turns the Poisson cohomology (4.16) into a graded commutative algebra. Similarly, the cup-product $\cup$ (3.13) turns the Hochschild cohomology (4.17) into a graded commutative algebra. Due to $[58,70]$ we have the following theorem

THEOREM 10. The isomorphism from the Poisson cohomology (4.16) to the Hochschild cohomology (4.17) induced by the $L_{\infty}$ quasi-isomorphism (4.15) is an isomorphism of graded commutative algebras.

This property was used in [19] by D. Calaque and M. Van den Bergh to prove Căldăraru's conjecture [22] on the Hochschild structure of an algebraic variety.

Shoikhet's quasi-isomorphism of $L_{\infty}$-modules [79] has a similar subtle compatibility property with the contraction $I$ (3.15). This property is proved by Calaque and Rossi [21]. In [21] the authors also applied this result to obtain a version of the Duflo isomorphism on coinvariants.

These results indicate that there should be a bridge between Kontsevich's construction [58] (resp. Shoikhet's construction [79]) and the construction of the second author [82] (resp. the construction in [39]). 


\subsection{FORMALITY THEOREMS FOR HOCHSCHILD AND CYCLIC COMPLEXES IN THE ALGEBRAIC GEOMETRY SETTING}

In the algebraic geometry setting, Theorem 4 has a formulation which does not need the amendment about the nature of cochains. In other words, we do not need to restrict ourselves to the polydifferential operators.

THEOREM 11. Let $X$ be a smooth affine variety over a field $\mathbb{K}$ of characteristic zero and $A=\mathcal{O}_{X}(X)$ be the algebra of regular functions on $X$. Then the $D G$ Lie algebra $\left(C^{\bullet+1}(A), \partial^{\text {Hoch }},[,]_{G}\right)$ of Hochschild cochains is quasi-isomorphic to the graded Lie algebra $\left(\wedge_{A}^{\bullet+1} \operatorname{Der}(A),[,]_{\mathrm{SN}}\right)$ of polyderivations of $A$.

This statement is an immediate corollary of Theorem 2 and Theorem 4 from [38]. It can be also extracted from Kontsevich's paper [60] on deformation quantization of algebraic varieties. However, Kontsevich's approach requires that the base field $\mathbb{K}$ contains the reals.

Beyond the affine case it no longer makes sense to talk about global sections. Thus we need to reformulate the question for the sheaves of Hochschild cochains.

According to Swan [81] and Yekutieli [95] an appropriate candidate for the sheaf of (normalized) Hochschild cochains on an arbitrary smooth algebraic variety $X$ is the sheaf of (normalized) polydifferential operators with regular coefficients. We denote this sheaf by $C^{\bullet}\left(\mathcal{O}_{X}\right)$. The Gerstenhaber bracket (3.14) equips $C^{\bullet+1}\left(\mathcal{O}_{X}\right)$ with the structure of a sheaf of DG Lie algebras.

Due to $[38,89,96]$ we have

THEOREM 12. For every smooth algebraic variety $X$ over a field $\mathbb{K}$ of characteristic zero the sheaf of $D G$ Lie algebra $C^{\bullet+1}\left(\mathcal{O}_{X}\right)$ is quasi-isomorphic to the sheaf $\wedge^{\bullet+1} T_{X}$ of polyvector fields with the Schouten-Nijenhuis bracket.

For applications of this theorem to deformation quantization in the setting of algebraic geometry we refer the reader to papers $[18,60,96]$.

Hochschild chains can also be added into this picture. An appropriate candidate for the sheaf of (normalized) Hochschild chains on an algebraic variety $X$ is the sheaf of (normalized) polyjets

$$
\text { C. }\left(\mathcal{O}_{X}\right)=\mathcal{H o m}_{\mathcal{O}_{X}}\left(C^{-\bullet}\left(\mathcal{O}_{X}\right), \mathcal{O}_{X}\right) \text {, }
$$

where $\mathcal{H o m}$ denotes the sheaf-Hom and $C^{\bullet}\left(\mathcal{O}_{X}\right)$ is considered with its natural left $\mathcal{O}_{X}$-module structure.

Using the isomorphism between $X$ and the main diagonal of the product $X^{\times k}$ we may identify local sections of $C_{k}\left(\mathcal{O}_{X}\right)$ with (normalized) $\infty$-jets on $X^{\times k}$ near its main diagonal.

To introduce sheaves of cyclic chains we introduce an auxiliary variable $u$ of degree 2 and consider a $\mathbb{K}[u]$-module $\mathcal{W}$ as a constant sheaf on $X$. Then to every 
such module we assign a sheaf of cyclic chains

$$
C C_{\bullet}^{\mathcal{W}}\left(\mathcal{O}_{X}\right)=\left(C_{\bullet}\left(\mathcal{O}_{X}\right)[[u]] \otimes_{\mathbb{K}[u]} \mathcal{W}, \partial^{\text {Hoch }}+u B\right),
$$

The operation $L$ (3.16) equips $C C_{\bullet}^{\mathcal{W}}\left(\mathcal{O}_{X}\right)$ with a structure of a sheaf of DG Lie algebra modules over the sheaf of DG Lie algebras $C^{\bullet}\left(\mathcal{O}_{X}\right)$.

Theorems 4 and 5 from [39] implies the following statement

THEOREM 13. Let $X$ be a smooth algebraic variety over a field $\mathbb{K}$ of characteristic zero. If $\mathcal{W}$ is a $\mathbb{R}[u]$-module of finite projective dimension then the pair "the sheaf of $D G$ Lie algebras $C^{\bullet+1}\left(\mathcal{O}_{X}\right)$ and the sheaf of its DG modules $C C_{\bullet}^{\mathcal{W}}\left(\mathcal{O}_{X}\right)$ " is quasiisomorphic to the pair "the sheaf of graded Lie algebras $\wedge^{\bullet+1} T_{X}$ and the sheaf of its $D G$ modules $\left(\Omega_{X}^{-\bullet}[[u]] \otimes_{\mathbb{R}[u]} \mathcal{W}, u d\right)$ ”.

Remark. Using the construction of M. Van den Bergh [89] and A. Yekutieli [95] as well as the results of B. Shoikhet [79] and T. Willwacher [94], it is possible to prove Theorem 13 under the assumption that the base field $\mathbb{K}$ contains the reals.

\section{More Applications of Formality Theorems}

Due to Corollary 1 the deformation class of a star product $*$ is uniquely determined by the Kontsevich class which is the equivalence class of a formal Poisson structure. For this reason the Kontsevich class is, sometimes, referred to as the characteristic class of a star-product. In many cases deformation quantization algebras are not obtained via formality theorems. In such situations it may be hard to find the Kontsevich class of the deformation quantization algebra. However, it is often possible to extract some information about representatives of this class using the homological properties of the deformation quantization algebra.

An example of this situation is provided by the unimodularity criterion from [35].

To describe this criterion we set $X$ to be a smooth affine variety with trivial canonical bundle. The triviality of the canonical bundle implies that there exists a nowhere vanishing top degree exterior form Vol on $X$.

A formal Poisson structure $\pi$ (4.6) is called unimodular [17,93] if there exists a formal power series

$$
\mathrm{Vol}_{\hbar}=\mathrm{Vol}+\sum_{k=1}^{\infty} \hbar^{k} \mathrm{Vol}_{k}
$$

of top degree exterior forms starting with a nowhere vanishing form Vol and such that

$$
l_{\pi} \mathrm{Vol}_{\hbar}=0,
$$

where $l_{\pi}$ is the Lie derivative (3.6). 
Let $A_{\hbar}$ be a deformation quantization algebra $(A[[\hbar]], *)$ of $X$. It turns out that $A_{\hbar}$ has Hochschild dimension equal to the dimension of $X$. Furthermore, $A_{\hbar}$ satisfies all the conditions of Theorem 3. Due to [35] we have the following homological unimodularity criterion:

THEOREM 14 [35, Theorem 3]. The Van den Bergh dualizing module

$$
V_{A_{\hbar}}=H H^{d}\left(A_{\hbar}, A_{\hbar} \otimes A_{\hbar}\right)
$$

of $A_{\hbar}=(A[[\hbar]], *)$ is isomorphic to $A_{\hbar}$ as a bimodule if and only if the formal Poisson structure $\pi$ (4.6) corresponding to the star-product $*$ is unimodular.

The proof of this criterion is based on the algebraic geometry version of Theorem 7. This criterion was used in a recent paper [41] by Etingof and Ginzburg to show that in a certain family of Calabi-Yau algebras associated to del Pezzo surfaces, each algebra has a non-trivial center.

Another application of formality theorems to quantization of unimodular Poisson structures is given in [27] by Cattaneo and Felder. In this paper they showed that if $\pi$ is a unimodular Poisson structure on a smooth real manifold $M$ then the deformation quantization algebra $\left(C^{\infty}(M)[[\hbar]], *\right)$ corresponding to $\pi$ admits the following trace functional

$$
f \mapsto \int_{M} f \operatorname{Vol}_{\hbar},
$$

where $f$ is a compactly supported function on $M$ and $\mathrm{Vol}_{\hbar}$ is a formal series of top degree forms starting with the volume form Vol satisfying the equation

$$
l_{\pi} \mathrm{Vol}=0 .
$$

Algebraic index theorems [37,85] for general Poisson manifolds give another tool for extracting information about the Kontsevich class of a star-product. These theorems express the isomorphism between Hochschild (respectively, periodic cyclic) homology of a deformation quantization algebra and the Poisson homology (respectively, de Rham cohomology) of the manifold or variety in terms of characteristic classes. For lack of space we do not give more details about these theorems here and instead refer the reader to $[37,85]$.

Many interesting examples of Poisson manifolds are obtained via reduction $[23,80]$. In papers [26,69] a "super"-version of Kontsevich's formality theorem is considered with the application to deformation quantization of reduced spaces in a fairly general situation. In both papers $[26,69]$ it was noticed that in general there may be obstructions to the construction of the star-product on the reduced space. This is not surprising because, in general, the reduction procedure gives a geometric object which is not even a manifold. The presence of this obstruction 
does not mean that certain reduced spaces should be discarded. It is rather an indication that our formulation of the quantization problem for such "spaces" should be modified.

The question of functoriality in deformation quantization is closely related to the above question on the reduction. We suspect that the ideas in [15] may shed some light on this question.

There are also very interesting applications of formality theorems for Hochschild complexes to Lie theory. It is Kontsevich's formality theorem [58] which helped to solve $[1-4,86]$ the long standing Kashiwara-Vergne conjecture [57]. Furthermore, using Kontsevich's formality theorem in [28] Cattaneo and Torossian generalized some of Lichnerowicz's results for the commutativity of the algebra of invariant differential operators and solved a long standing problem posed by Duflo for the expression of invariant differential operators on any symmetric spaces in exponential coordinates. They also developed a new method to construct characters for algebras of invariant differential operators.

\section{An Example of a Non-Formal DG Lie Algebra}

Let $A=\mathbb{K}\left[x^{1}, \ldots, x^{d}\right]$ be a polynomial algebra in $d$ variables over $\mathbb{K}$. It is obvious that the DG Lie algebra structure on $C^{\bullet+1}(A)$ restricts to the truncated Hochschild complex $C^{\geq 1}(A)$ of $A$. Furthermore, due to the commutativity of $A$, the Hochschild differential vanishes on the degree zero cochains. Therefore, the cohomology of the truncated Hochschild complex $C^{\geq 1}(A)$ is the vector space

$$
\wedge^{\geq 1} \operatorname{Der}(A)
$$

of polynomial polyvector fields on $\mathbb{K}^{d}$ of degrees $\geq 1$.

In this subsection we prove that

THEOREM 15. If $d$ is even then the DG Lie algebra $C^{\geq 1}(A)$ is non-formal.

Remark 1. This theorem answers a question of the referee of our paper [38].

Remark 2. We suspect that the case of odd dimension can be considered similarly with the help of a regular constant Poisson structure of maximal rank.

Proof (by contradiction). The formality of the DG Lie algebra $C^{\geq 1}(A)$ would still imply a bijection between the equivalence classes of star-products and the equivalence classes of formal Poisson structures on the affine space $\mathbb{K}^{d}$.

Let

$$
F: \wedge^{\geq 1} \operatorname{Der}(A) \leadsto C^{\geq 1}(A)
$$


be an $L_{\infty}$ quasi-isomorphism from the graded Lie algebra $\wedge^{\geq 1} \operatorname{Der}(A)$ to the DG Lie algebra $C^{\geq 1}(A)$.

As we mentioned in Section 2 the structure map

$$
F_{1}: \wedge^{\geq 1} \operatorname{Der}(A) \rightarrow C^{\geq 1}(A)
$$

is a quasi-isomorphism of cochain complexes.

In general, this quasi-isomorphism may differ from the standard HochschildKostant-Rosenberg inclusion

$$
I_{\mathrm{HKR}}: \wedge^{\geq 1} \operatorname{Der}(A) \hookrightarrow C^{\geq 1}(A) .
$$

by a coboundary term.

If this coboundary term is non-zero then applying Lemma 1 from [36] we modify the $L_{\infty}$ quasi-isomorphism (6.1) in such a way that $F_{1}$ will coincide with (6.3). Thus, we may assume, without loss of generality, that

$$
F_{1}=I_{\mathrm{HKR}} .
$$

The correspondence between the formal Poisson structures (4.6) and the star-products is given by the assignment

$$
\begin{aligned}
\pi & \mapsto * \\
a * b & =a b+\sum_{k=1}^{\infty} \frac{1}{k !} F_{k}(\pi, \pi, \ldots, \pi)(a, b) .
\end{aligned}
$$

Furthermore, $\pi$ allows us to twist the quasi-isomorphism (6.1) to the $L_{\infty}$ morphism

$$
F^{\pi}: \wedge^{\geq 1} \operatorname{Der}(A)[[\hbar]] \sim C^{\geq 1}\left(A_{\hbar}\right),
$$

where $A_{\hbar}$ is the algebra $A[[\hbar]]$ with the star-product (6.6) and the DG Lie algebra $\wedge^{\geq 1} \operatorname{Der}(A)[[\hbar]]$ is considered with the Lichnerowicz differential $[\pi,]_{\mathrm{SN}}$.

According to [34] or [46] the structure maps of the twisted $L_{\infty}$ quasi-isomorphism $F^{\pi}$ are given by the formula

$$
F_{n}^{\pi}\left(\gamma_{1}, \ldots, \gamma_{n}\right)=\sum_{k=0}^{\infty} \frac{1}{k !} F_{n+k}\left(\pi, \pi, \ldots, \pi, \gamma_{1}, \gamma_{2}, \ldots, \gamma_{n}\right) .
$$

An obvious analog of Proposition 1 allows us to conclude that the structure map of the first level

$$
F_{1}^{\pi}:\left(\wedge^{\geq 1} \operatorname{Der}(A)[[\hbar]],[\pi,]_{\mathrm{SN}}\right) \stackrel{\sim}{\rightarrow} C^{\geq 1}\left(A_{\hbar}\right)
$$

is a quasi-isomorphism of cochain complexes. 
Thus localizing (6.6) in $\hbar$ we get the following $L_{\infty}$ quasi-isomorphism of DG Lie algebras

$$
F^{\pi}:\left(\wedge^{\geq 1} \operatorname{Der}(A)((\hbar)),[\pi,]_{\mathrm{SN}}\right) \sim C^{\geq 1}\left(A_{\hbar}\left[\hbar^{-1}\right]\right) .
$$

The 0th cohomology of the DG Lie algebra $\left(\wedge^{\geq 1} \operatorname{Der}(A)((\hbar)),[\pi,]_{\mathrm{SN}}\right)$ is the Lie algebra of Poisson vector fields and the 0th cohomology of the DG Lie algebra $C^{\geq 1}\left(A_{\hbar}\left[\hbar^{-1}\right]\right)$ is the Lie algebra of derivations of $A_{\hbar}\left[\hbar^{-1}\right]$.

Therefore, the $L_{\infty}$ quasi-isomorphism (6.9) gives us an isomorphism from the Lie algebra of Poisson vector fields of $\pi$ to the Lie algebra $\operatorname{Der}\left(A_{\hbar}\left[\hbar^{-1}\right]\right)$ of derivations of $A_{\hbar}\left[\hbar^{-1}\right]$.

Since $d$ is even we may choose $\pi=\hbar \theta$ where $\theta$ is a non-degenerate constant bivector.

Let us denote by $*_{W}$ the Moyal-Weyl star-product which quantizes $\theta$

$$
a *_{W} b=\left.\exp \left(\frac{\hbar}{2} \theta^{i j} \frac{\partial}{\partial x^{i}} \frac{\partial}{\partial y^{j}}\right) a(x) b(y)\right|_{x=y} .
$$

Due to (6.4) the equivalence class of $*_{W}$ corresponds to an equivalence class of a formal Poisson structure $\tilde{\pi}$ which starts with $\hbar \theta$ :

$$
\tilde{\pi}=\hbar \theta+\hbar^{2} \widetilde{\pi}_{2}+\hbar^{3} \widetilde{\pi}_{3}+\cdots .
$$

Since $\theta$ is a non-degenerate bivector the formal Poisson structure $\tilde{\pi}$ is equivalent to the original Poisson structure $\pi=\hbar \theta$. This statement can be easily deduced from the fact that, in the symplectic case, the Poisson cohomology is isomorphic to the de Rham cohomology.

Therefore, the Moyal-Weyl star-product $*_{W}$ is equivalent to $*(6.6)$. Hence the Lie algebra $\operatorname{Der}\left(A_{\hbar}{ }^{W}\right)$ of derivations of the Weyl algebra

$$
A_{\hbar}{ }^{W}=\left(A((\hbar)), *_{W}\right)
$$

is isomorphic to the Lie algebra of Poisson vector fields of $\hbar \theta$.

It is known that the Lie algebra $\operatorname{Der}\left(A_{\hbar}{ }^{W}\right)$ of derivations of the Weyl algebra $A_{\hbar}{ }^{W}$ is isomorphic to

$$
\left((A / \mathbb{K})((\hbar)),[,]_{*_{W}}\right),
$$

where $[a, b]_{*_{W}}=a *_{W} b-b *_{W} a$. The desired isomorphism is defined by assigning to an element $a \in(A / \mathbb{K})((\hbar))$ the corresponding inner derivation:

$$
a \mapsto[a,]_{*_{W}} .
$$

Here we identify the quotient $A / \mathbb{K}$ with the ideal of polynomials vanishing at the origin.

Similarly, the Lie algebra of Poisson vector fields of $\hbar \theta$ is isomorphic to

$$
\left((A / \mathbb{K})((\hbar)),\{,\}_{\hbar \theta}\right),
$$


where $\{a, b\}_{\hbar \theta}=\hbar i_{\theta} d a d b$. The desired isomorphism is defined by assigning to an element $a \in(A / \mathbb{K})((\hbar))$ the corresponding Hamiltonian vector field:

$$
a \mapsto\{a,\}_{\hbar \theta} .
$$

Thus we conclude that an $L_{\infty}$ quasi-isomorphism $F$ (6.1) would give us an isomorphism $T$ from the Lie algebra (6.14) to the Lie algebra (6.13) over the field $\mathbb{K}((\hbar))$. Furthermore, Equations (6.4) and (6.7) imply that this isomorphism $T$ satisfies the following property

$$
T(a)=a \bmod \hbar, \quad \forall a \in A / \mathbb{K} .
$$

The Lie bracket in (6.13) has the form

$$
[a, b]_{*_{W}}=\hbar \theta^{i j} \partial_{x_{i}} a \partial_{x^{j}} b+\hbar^{3} V(a, b) \bmod \hbar^{5},
$$

where

$$
\begin{aligned}
& V(a, b)=\frac{1}{24} \theta_{1}^{i_{1} j_{1}} \theta_{1}^{i_{2} j_{2}} \theta_{1}^{i_{3} j_{3}} \partial_{x^{i_{1}}} \partial_{x^{i_{2}}} \partial_{x^{i_{3}}} a \partial_{x^{j_{1}}} \partial_{x^{j_{2}}} \partial_{x^{j_{3}}} b, \\
& a, b \in(A / \mathbb{K})((\hbar)) .
\end{aligned}
$$

From deformation theory it follows that the map

$$
V: A / \mathbb{K} \otimes A / \mathbb{K} \rightarrow A / \mathbb{K}
$$

given by the formula (6.17) is a cocycle for the Lie algebra $A / \mathbb{K}$ with the bracket

$$
\{a, b\}=\theta^{i j} \partial_{x^{i}} a \partial_{x^{j}} b .
$$

The existence of the isomorphism $T$ satisfying the property (6.15) would imply that this cocycle is trivial. In other words, there should exist a linear map

$$
P: A / \mathbb{K} \rightarrow A / \mathbb{K}
$$

such that

$$
\begin{aligned}
V(a, b) & =P(\{a, b\})-\{P(a), b\}-\{a, P(b)\}, \\
a, b & \in A / \mathbb{K} .
\end{aligned}
$$

It is not hard to see that if the cocycle $V(6.17)$ is trivial in the general case then it is trivial in the two-dimensional case with the canonical Poisson bracket

$$
\begin{aligned}
\{a, b\}(x, y) & =\partial_{x} a(x, y) \partial_{y} b(x, y)-\partial_{y} a(x, y) \partial_{x} b(x, y), \\
a, b & \in \mathbb{K}[x, y] / \mathbb{K} .
\end{aligned}
$$

Thus we may restrict ourselves to the two-dimensional case with the canonical Poisson bracket (6.20). 
In this case the cocycle $V$ reads

$$
\begin{aligned}
V(a, b) & =\frac{1}{24}\left(\partial_{x}^{3}(a) \partial_{y}^{3} b-3 \partial_{x}^{2} \partial_{y}(a) \partial_{x} \partial_{y}^{2}(b)+3 \partial_{x} \partial_{y}^{2}(a) \partial_{x}^{2} \partial_{y}(b)-\partial_{y}^{3}(a) \partial_{x}^{3}(b)\right), \\
a, b & \in \mathbb{K}[x, y] / \mathbb{K} .
\end{aligned}
$$

For $a=x$ and $b=y$ Equation (6.19) implies that

$$
\partial_{x} P(x)+\partial_{y} P(y) \in \mathbb{K}
$$

Let $c_{0}=\frac{1}{2}\left(\partial_{x} P(x)+\partial_{y} P(y)\right)$. Then, setting $Q_{1}=P(x)-c_{0} x$ and $Q_{2}=P(y)-c_{0} y$, we get

$$
\partial_{x} Q_{1}+\partial_{y} Q_{2}=0
$$

Hence, there exists a polynomial $Q \in \mathbb{K}[x, y]$ such that $Q_{1}=\{Q, x\}$ and $Q_{2}=\{Q, y\}$.

Thus, adjusting $P$ by a Hamiltonian vector field, we reduce it to the form in which

$$
P(x)=c_{0} x, \quad P(y)=c_{0} y .
$$

Substituting in Equation (6.19) quadratic monomials for $a$ and linear for $b$ we deduce that

$$
\begin{aligned}
& P\left(x^{2}\right)=c_{11} x+c_{12} y, \\
& P(x y)=c_{21} x+c_{22} y, \\
& P\left(y^{2}\right)=c_{31} x+c_{32} y,
\end{aligned}
$$

where $c_{i j} \in \mathbb{K}$. Next substituting quadratic monomials for $a$ and $b$ we deduce that $c_{12}=c_{31}=0, c_{11}=2 c_{22}$ and $c_{32}=2 c_{21}$. Thus

$$
\begin{aligned}
& P\left(x^{2}\right)=2 c_{y} x, \\
& P(x y)=c_{x} x+c_{y} y, \\
& P\left(y^{2}\right)=2 c_{x} y
\end{aligned}
$$

for some constants $c_{x}, c_{y} \in \mathbb{K}$.

Adjusting $P$ by the Hamiltonian vector field

$$
\left\{c_{x} x-c_{y} y,\right\}
$$

we kill the right-hand sides in (6.23).

Thus we may assume that

$$
\begin{gathered}
P(x)=c_{0} x, \quad P(y)=c_{0} y, \\
P\left(x^{2}\right)=P(x y)=P\left(y^{2}\right)=0 .
\end{gathered}
$$


Next, plugging in cubic monomials $x^{3}, x^{2} y, x y^{2}, y^{3}$ for $a$ and linear monomials for $b$ in (6.19), we deduce that

$$
\begin{aligned}
P\left(x^{3}\right) & =b_{11} x+b_{12} y-c_{0} x^{3}, \\
P\left(x^{2} y\right) & =b_{21} x+b_{22} y-c_{0} x^{2} y, \\
P\left(x y^{2}\right) & =b_{31} x+b_{32} y-c_{0} x y^{2}, \\
P\left(y^{3}\right) & =b_{41} x+b_{42} y-c_{0} y^{3},
\end{aligned}
$$

where $b_{i j} \in \mathbb{K}$.

To get a further restriction we substitute cubic monomials for $a$ and quadratic for $b$ in (6.19). We get that all the coefficients $b_{i j}$ should vanish. Thus

$$
\begin{aligned}
P\left(x^{3}\right) & =-c_{0} x^{3}, \quad P\left(x^{2} y\right)=-c_{0} x^{2} y, \\
P\left(x y^{2}\right) & =-c_{0} x y^{2}, \quad P\left(y^{3}\right)=-c_{0} y^{3},
\end{aligned}
$$

Every quartic monomial can be written as a Poisson bracket of two cubic monomials. Using this observation and Equation (6.19) for $a$ and $b$ being cubic monomials we deduce that

$$
P\left(x^{n} y^{k}\right)=-2 c_{0} x^{n} y^{k}
$$

whenever $n+k=4$.

To get the desired contradiction, we first set $a=x^{4}$ and $b=y^{3}$ in (6.19) and get

$$
6 x-\left(12 P\left(x^{3} y^{2}\right)+2 c_{0}\left\{x^{4}, y^{3}\right\}+c_{0}\left\{x^{4}, y^{3}\right\}\right) \in \mathbb{K}
$$

or equivalently

$$
12 P\left(x^{3} y^{2}\right)-6 x+3 c_{0} x^{3} y^{2} \in \mathbb{K} .
$$

Secondly, plugging $a=x^{3} y$ and $b=x y^{2}$ into (6.19) we get

$$
-\frac{3}{2} x-\left(5 P\left(x^{3} y^{2}\right)+2 c_{0}\left\{x^{3} y, x y^{2}\right\}+c_{0}\left\{x^{3} y, x y^{2}\right\}\right)
$$

or equivalently

$$
5 P\left(x^{3} y^{2}\right)-\frac{3}{2} x+3 c_{0} x^{3} y^{2} \in \mathbb{K} .
$$

The inclusion in (6.27) clearly contradicts the inclusion in (6.28) and the theorem follows.

Remark 1. The same expression (6.17) defines a cocycle for the Lie algebra $A$ with the bracket $\{,\}_{\theta}$. It was shown by Vey [90] that this cocycle is non-trivial. Here we had to work with the quotient $A / \mathbb{K}$ and this is why we had to redo the computation of Vey taking into account this modification. 
Remark 2. It makes sense to consider a modification of the Weyl algebra which is defined over $\mathbb{K}$. This is the algebra $A^{W}$ generated by $x^{i}$ 's satisfying the relations

$$
x^{i} x^{j}-x^{j} x^{i}=\theta^{i j}
$$

where $\theta^{i j}$ is as above a non-degenerate antisymmetric constant matrix. It is known [9] that the Lie algebra of derivations of $A^{W}$ is not isomorphic to the Lie algebra of derivations of the corresponding Poisson algebra $\mathbb{K}\left[x^{1}, \ldots, x^{d}\right]$ with the bracket $\{,\}_{\theta}$. This fact is mentioned in [9] as negative evidence for the conjecture about the automorphisms of the Weyl algebra.

\section{Acknowledgements}

We would like to thank J. Stasheff for discussions and for his useful comments on the first version of our manuscript. D.T. and B.T. are supported by NSF grants. The work of V.D. is partially supported by the Grant for Support of Scientific Schools NSh-3036.2008.2.

Open Access This article is distributed under the terms of the Creative Commons Attribution Noncommercial License which permits any noncommercial use, distribution, and reproduction in any medium, provided the original author(s) and source are credited.

\section{References}

1. Alekseev, A., Meinrenken, E.: On the Kashiwara-Vergne conjecture. Invent. Math. 164, 615-634 (2006)

2. Andler, M., Dvorsky, A., Sahi, S.: Deformation quantization and invariant distributions. C. R. Acad. Sci. Paris Sér. I Math. 330(2), 115-120 (2000)

3. Andler, M., Dvorsky, A., Sahi, S.: Kontsevich quantization and invariant distributions on Lie groups. Ann. Sci. Ecole Norm. Sup. (4) 35(3), 371-390 (2002)

4. Andler, M., Sahi, S., Torossian, C.: Convolution of invariant distributions: proof of the Kashiwara-Vergne conjecture. Lett. Math. Phys. 69, 177-203 (2004)

5. Arnal, D., Manchon, D., Masmoudi, M.: Choix des signes pour la formalité de M. Kontsevich. Pac. J. Math. 203(1), 23-66 (2002)

6. Babenko, I.K., Taimanov, I.A.: Massey products in symplectic manifolds. Sb. Math. 191(7-8), 1107-1146 (2000)

7. Bayen, F., Flato, M., Fronsdal, C., Lichnerowicz, A., Sternheimer, D.: Deformation theory and quantization. I. Deformations of symplectic structures. Ann. Phys. (N.Y.) 111, 61-110 (1978)

8. Bayen, F., Flato, M., Fronsdal, C., Lichnerowicz, A., Sternheimer, D.: Deformation theory and quantization, II. Physical applications. Ann. Phys. (N.Y.) 111, 111-151 (1978)

9. Belov-Kanel, A., Kontsevich, M.: Automorphisms of the Weyl algebra. Lett. Math. Phys. 74(2), 181-199 (2005). arXiv:math/0512169

10. Berezin, F.A.: Quantization. Izv. Akad. Nauk. 38, 1116-1175 (1974)

11. Berezin, F.A.: General concept of quantization. Commun. Math. Phys. 40, 153-174 (1975) 
12. Berger, C., Fresse, B.: Combinatorial operad actions on cochains. Math. Proc. Camb. Philos. Soc. 137(1), 135-174 (2004)

13. Berger, C., Moerdijk, I.: Axiomatic homotopy theory for operads. Comment. Math. Helv. 78(4), 805-831 (2003). arXiv:math/0206094

14. Boardmann, J.M., Vogt, R.M.: Homotopy Invariant Algebraic Structures on Topological Spaces. Lecture Notes in Mathematics, vol. 347. Springer, Berlin (1973)

15. Borisov, D.V.: $G_{\infty}$-structure on the deformation complex of a morphism. J. Pure Appl. Algebra 210(3), 751-770 (2007)

16. Brylinski, J.-L.: A differential complex for Poisson manifolds. J. Differ. Geom. 28(1), 93-114 (1988)

17. Brylinski, J.-L., Zuckerman, G.: The outer derivation of a complex Poisson manifold. J. Reine Angew. Math. 506, 181-189 (1999)

18. Calaque, D., Halbout, G.: Weak quantization of Poisson structures. arXiv:0707.1978

19. Calaque, D., Van den Bergh, M.: Hochschild cohomology and Atiyah classes. arXiv:0708.2725

20. Calaque, D., Van den Bergh, M.: Global formality at the $G_{\infty}$-level. arXiv:0710.4510

21. Calaque, D., Rossi, C.A.: Shoikhet's conjecture and Duflo isomorphism on (Co)invariants. SIGMA 4, 060 (2008). arXiv:0805.2409

22. Căldăraru, A.: The Mukai pairing. II. The Hochschild-Kostant-Rosenberg isomorphism. Adv. Math. 194(1), 34-66 (2005)

23. Cattaneo, A.S.: Deformation quantization and reduction. In: Poisson Geometry in Mathematics and Physics. Contemporary Mathematics, vol. 450, pp. 79-101. American Mathematical Society, Providence (2008)

24. Cattaneo, A.S., Felder, G.: A path integral approach to the Kontsevich quantization formula. Commun. Math. Phys. 212(3), 591-611 (2000), arXiv:math/9902090

25. Cattaneo, A.S., Felder, G., Tomassini, L.: From local to global deformation quantization of Poisson manifolds. Duke Math. J. 115(2), 329-352 (2002). math.QA/0012228

26. Cattaneo, A.S., Felder, G.: Relative formality theorem and quantisation of coisotropic submanifolds. Adv. Math. 208(2), 521-548 (2007)

27. Cattaneo, A.S., Felder, G.: Effective Batalin-Vilkovisky theories, equivariant configuration spaces and cyclic chains. Progr. Math. (2009, in press). arXiv:0802.1706

28. Cattaneo, A.S., Torossian, C.: Quantification pour les paires symetriques et diagrammes de Kontsevich. Ann. Sci. Ec. Norm. Sup. 41, 789-854 (2008). arXiv:math/0609693

29. Cuntz, J., Skandalis, G., Tsygan, B.: Cyclic homology in non-commutative geometry. In: Encyclopaedia of Mathematical Sciences, vol. 121. Operator Algebras and Non-commutative Geometry, II. Springer, Berlin (2004)

30. Daletski, Yu., Gelfand, I., Tsygan, B.: On a variant of noncommutative geometry. Sov. Math. Dokl. 40(2), 422-426 (1990)

31. Deligne, P., Griffiths, P., Morgan, J., Sullivan, D.: Real homotopy theory of Kähler manifolds. Invent. Math. 29(3), 245-274 (1975)

32. Dolgushev, V.A.: Covariant and Equivariant Formality Theorems. Adv. Math. 191(1), 147-177 (2005). arXiv:math/0307212

33. Dolgushev, V.A.: A Formality Theorem for Hochschild Chains. Adv. Math. 200(1), 51-101 (2006). math.QA/0402248

34. Dolgushev, V.A.: A Proof of Tsygan's formality conjecture for an arbitrary smooth manifold. PhD thesis, MIT. math.QA/0504420

35. Dolgushev, V.A.: The Van den Bergh duality and the modular symmetry of a Poisson variety. Selecta Math. (N.S.) 14(2), 199-228 (2009). arXiv:math/0612288 
36. Dolgushev, V.A.: Erratum to: "A Proof of Tsygan's Formality Conjecture for an Arbitrary Smooth Manifold." arXiv:math/0703113

37. Dolgushev, V.A., Rubtsov, V.N.: An algebraic index theorem for Poisson manifolds. J. Reine Angew. Math. (Crelles J.) 2009(633), 77-113 (2009)

38. Dolgushev, V., Tamarkin, D., Tsygan, B.: The homotopy Gerstenhaber algebra of Hochschild cochains of a regular algebra is formal. J. Noncommut. Geom. 1(1), 1-25 (2007). arXiv:math/0605141

39. Dolgushev, V.A., Tamarkin, D.E., Tsygan, B.L.: Formality of the homotopy calculus algebra of Hochschild (co)chains. arXiv:0807.5117

40. Drinfeld, V.G.: Quasi-Hopf algebras. Leningr. Math. J. 1(6), 1419-1457 (1990)

41. Etingof, P., Ginzburg, V.: Noncommutative del Pezzo surfaces and Calabi-Yau algebras. arXiv:0709.3593

42. Gelfand, I.M., Fuchs, D.V.: Cohomology of the algebra of formal vector fields. Izv. Akad. Nauk., Math. Ser. (In Russian) 34, 322-337 (1970)

43. Gelfand, I.M., Kazhdan, D.A.: Some problems of differential geometry and the calculation of cohomologies of Lie algebras of vector fields. Sov. Math. Dokl. 12(5), 1367-1370 (1971)

44. Gerstenhaber, M.: The cohomology structure of an associative ring. Ann. Math. 78, 267-288 (1963)

45. Getzler, E.: A Darboux theorem for Hamiltonian operators in the formal calculus of variations. Duke Math. J. 111(3), 535-560 (2002)

46. Getzler, E.: Lie theory for nilpotent L-infinity algebras. Ann. Math. 170(1), 271-301 (2009). arXiv:math/0404003

47. Getzler, E., Jones, J.D.S.: Operads, homotopy algebra and iterated integrals for double loop spaces. hep-th/9403055

48. Ginzburg, V.: Calabi-Yau algebras. math.AG/0612139

49. Ginzburg, V., Kapranov, M.: Koszul duality for operads. Duke Math. J. 76(1), 203-272 (1944)

50. Goldman, W., Millson, J.: The deformation theory of representation of fundamental groups in compact Kähler manifolds. Publ. Math. I.H.E.S. 67, 43-96 (1988)

51. Halperin, S., Stasheff, J.: Obstructions to homotopy equivalences. Adv. Math. 32(3), 233-279 (1979)

52. Henriques, A.: Integrating $L_{\infty}$-algebras. Compos. Math. 144(4), 1017-1045 (2008)

53. Hinich, V.: Homological algebra of homotopy algebras. Comm. Algebra 25(10), 3291-3323 (1997)

54. Hinich, V.: Tamarkin's proof of Kontsevich formality theorem. Forum Math. 15(4), 591-614 (2003). math.QA/0003052

55. Hinich, V., Schechtman, V.: Homotopy Lie algebras. I.M. Gelfand Seminar. Adv. Sov. Math. 16(2), 1-28 (1993)

56. Ikeda, N.: Two-dimensional gravity and nonlinear gauge theory. Ann. Phys. 235, 435-464 (1994)

57. Kashiwara, M., Vergne, M.: The Campbell-Hausdorff formula and invariant hyperfunctions. Invent. Math. 47, 249-272 (1978)

58. Kontsevich, M.: Deformation quantization of Poisson manifolds. Lett. Math. Phys. 66, 157-216 (2003). q-alg/9709040

59. Kontsevich, M.: Formality conjecture. In: Sternheimer, D., et al. (eds.) Deformation Theory and Symplectic Geometry, pp. 139-156. Kluwer, Dordrecht (1997)

60. Kontsevich, M.: Deformation quantization of algebraic varieties. Moshé Flato memorial conference 2000, Part III (Dijon). Lett. Math. Phys. 56(3), 271-294 (2001) 
61. Kontsevich, M.: Operads and motives in deformation quantization. Lett. Math. Phys. 48, 35-72 (1999)

62. Kontsevich, M., Soibelman, Y.: Deformations of algebras over operads and the Deligne conjecture. In: Proceedings of the Moshé Flato Conference. Mathematical Physics Studies, vol. 21, pp. 255-307, Kluwer, Dordrecht (2000)

63. Kontsevich, M., Soibelman, Y.: Notes on A-infinity algebras, A-infinity categories and non-commutative geometry. I. math.RA/0606241

64. Koszul, J.L.: Crochet de Schouten-Nijenhuis et cohomologie, Astérisque (1985). Numéro Hors Série, pp. 257-271

65. Lada, T., Stasheff, J.: Introduction to SH Lie algebras for physicists. Int. J. Theor. Phys. 32(7), 1087-1103 (1993)

66. Lambrechts, P., Volic, I.: Formality of the little N-disks operad. arXiv:0808.0457

67. Lichnerowicz, A.: Les variétés de Poisson et leurs algèbres de Lie associées. J. Differ. Geom. 12(2), 253-300 (1977)

68. Loday, J.-L.: Cyclic Homology, Grundlehren der mathematischen Wissenschaften, vol. 301. Springer, Berlin (1992)

69. Lyakhovich, S.L., Sharapov, A.A.: BRST theory without Hamiltonian and Lagrangian. J. High Energy Phys. 3(011), 22 (2005)

70. Manchon, D., Torossian, C.: Cohomologie tangente et cup-produit pour la quantification de Kontsevich. Ann. Math. Blaise Pascal 10(1), 75-106 (2003)

71. Markl, M.: Models for operads. Comm. Algebra 24, 1471-1500 (1996)

72. May, J.P.: Infinite loop space theory. Bull. Am. Math. Soc. 83(4), 456-494 (1977)

73. May, J.P.: Matrix Massey products. J. Algebra 12, 533-568 (1969)

74. McClure, J.E., Smith, J.H.: A solution of Deligne's Hochschild cohomology conjecture. In: Recent Progress in Homotopy Theory (Baltimore, MD, 2000). Contemporary Mathematics, vol. 293, pp. 153-193. American Mathematical Society, Providence (2002). math.QA/9910126

75. Quillen, D.: Rational homotopy theory. Ann. Math. 90(2), 205-295 (1969)

76. Schaller, P., Strobl, T.: Poisson structure induced (topological) field theories. Modern Phys. Lett. A 9(33), 3129-3136 (1994)

77. Schlessinger, M., Stasheff, J.: Deformation theory and rational homotopy type, University of North Carolina preprint (1979)

78. Schlessinger, M., Stasheff, J.: The Lie algebra structure of tangent cohomology and deformation theory. J. Pure Appl. Algebra 38(2-3), 313-322 (1985)

79. Shoikhet, B.: A proof of the Tsygan formality conjecture for chains. Adv. Math. 179(1), 7-37 (2003). math.QA/0010321.

80. Stasheff, J.: Homological reduction of constrained Poisson algebras. J. Differ. Geom. 45, 221-240 (1997)

81. Swan, R.G.: Hochschild cohomology of quasiprojective schemes. J. Pure Appl. Algebra 110(1), 57-80 (1996)

82. Tamarkin, D.: Another proof of M. Kontsevich formality theorem. math.QA/9803025

83. Tamarkin, D.: Formality of chain operad of little discs. Lett. Math. Phys. 66(1-2), 65-72 (2003). math.QA/9809164

84. Tamarkin, D.: What do DG categories form? Compos. Math. 143(5), 1335-1358 (2007). math.CT/0606553

85. Tamarkin, D., Tsygan, B.: Cyclic formality and index theorems, Talk given at the Moshé Flato Conference (2000). Lett. Math. Phys. 56(2), 85-97 (2001)

86. Torossian, C.: Sur la conjecture combinatoire de Kashiwara-Vergne. J. Lie Theory 12(2), 597-616 (2002) 
87. Tsygan, B.: Formality conjectures for chains. In: Differential Topology, Infinite-dimensional Lie Algebras, and Applications. American Mathematical Society Translations, Series 2, vol. 194, pp. 261-274. American Mathematical Society, Providence (1999)

88. Van den Bergh, M.: A relation between Hochschild homology and cohomology for Gorenstein rings. Proc. Am. Math. Soc. 126(5), 1345-1348 (1998); Erratum to "A Relation between Hochschild Homology and Cohomology for Gorenstein Rings." Proc. Am. Math. Soc. 130(9), 2809-2810 (2002)

89. Van den Bergh, M.: On global deformation quantization in the algebraic case. J. Algebra 315(1), 326-395 (2007)

90. Vey, J.: Déformation du crochet de Poisson sur une variété symplectique. Comment. Math. Helv. 50, 421-454 (1975)

91. Voronov, A.: Quantizing Poisson manifolds. In: Perspectives on Quantization (South Hadley, MA, 1996). Contemporary Mathematics, vol. 214, pp. 189-195. American Mathematical Society, Providence (1998)

92. Voronov, A.A.: Homotopy Gerstenhaber algebras. In: Proceedings of the Moshé Flato Conference. Mathematical Physics Studies, vol. 22, pp. 307-331. Kluwer, Dordrecht (2000)

93. Weinstein, A.: The modular automorphism group of a Poisson manifold. J. Geom. Phys. 23(3-4), 379-394 (1997)

94. Willwacher, T.: Formality of cyclic chains. arXiv:0804.3887

95. Yekutieli, A.: The continuous Hochschild cochain complex of a scheme. Can. J. Math. 54(6), 1319-1337 (2002)

96. Yekutieli, A.: Deformation quantization in algebraic geometry. Adv. Math. 198(1), 383-432 (2005). math.AG/0310399 